Barriga, F.J.A.S., Binns, R.A., Miller, D.J., and Herzig, P.M. (Eds.)

Proceedings of the Ocean Drilling Program, Scientific Results Volume 193

\section{DATA REPORT: SUMmARY OF REVISED Alteration Phases for paCmanUs HYDROTHERMAL FIELD-X-RAY Diffraction Analyses of Altered Felsic VOLCANIC ROCKS FROM HOLES 1188A, 1188F, 1189A, AND 1189B'}

Klas S. Lackschewitz, ${ }^{2,3}$ Ryuji Asada, ${ }^{4}$ and Holger Paulick ${ }^{5}$
${ }^{1}$ Lackschewitz, K.S., Asada, R., and Paulick, H., 2006. Data report: Summary of revised alteration phases for PACMANUS hydrothermal field$\mathrm{X}$-ray diffraction analyses of altered felsic volcanic rocks from Holes 1188A, 1188F, 1189A, and 1189B. In Barriga, F.J.A.S., Binns, R.A., Miller, D.J., and Herzig, P.M. (Eds.), Proc. ODP, Sci. Results, 193, 1-19 [Online]. Available from World Wide Web: $<$ http://www-odp.tamu.edu/ publications/193_SR/VOLUME/ CHAPTERS/213.PDF>. [Cited YYYYMM-DD]

${ }^{2}$ Fachbereich Geowissenschaften, Universität Bremen, Klagenfurter Strasse 2, 28334 Bremen, Germany. klackschewitz@ifm-geomar.de ${ }^{3}$ Leibniz-Institut für

Meereswissenschaften, IFM-GEOMAR, Wischhofstrasse 1-3, 24124 Kiel, Germany.

${ }^{4}$ Department of Earth Sciences, Faculty of Science, Kanazawa University, Kakuma, Kanazawa, Ishikawa Prefecture 920-1192, Japan ${ }^{5}$ Mineralogisch-Petrologisches Institut, Universität Bonn, Poppelsdorfer Schloss, 53115 Bonn, Germany.

Initial receipt: 4 November 2003 Acceptance: 5 February 2006 Web publication: 5 July 2006 Ms 193SR-213 
and the active New Britain Trench to the south (Fig. F1). The eastern Manus Basin is characterized by a series of isolated hydrothermal fields including the PACMANUS hydrothermal field located near the crest of the 500- to 700-m-high Pual Ridge.

In this report we present the results of postcruise X-ray diffraction analyses of whole-rock samples from two sites analyzed by three different principal investigators. The sites can be separated into two groups based on their geographic locality. Site 1188 was drilled in the Snowcap hydrothermal site, representing a diffuse venting area, and Site 1189 was drilled in the Roman Ruins site, containing numerous actively high-temperature venting chimneys (Fig. F2). Hydrothermal alteration varies with depth and is complicated by overprinting relationships. The main alteration features are summarized in Binns, Barriga, Miller, et al. (2002).

\section{METHODS}

Samples analyzed in this study are from Holes $1188 \mathrm{~A}$ and $1188 \mathrm{~F}$ at Snowcap and 1189A and 1189B at Roman Ruins.

After air-drying, whole-rock mineralogy was determined by X-ray diffractometry. For mineral identification of powered samples from principal investigator K. Lackschewitz, a Philips X-ray diffractometer PW 1710 with monochromatic $\mathrm{CuK} \alpha$ was used. Qualitative phase identification was performed using MacDiff software (version 4.2.5) from R. Petschick (servermac.geologie.uni-frankfurt.de/Staff/Homepages/ Petschick/RainerE.html).

Another set of air-dried powdered samples from principal investigator H. Paulick was analyzed at the XRD Laboratory of the Mineralogical Institute, Freiberg University of Mining and Technology (Germany). Here, a Seifert-Freiberger Präzisionsmechanik GmbH RD7 instrument was used to generate X-ray diffractograms applying monochromatic $\mathrm{CuK} \alpha$ radiation. Qualitative phase identification was performed using Seifert-Analyze software.

Principal investigator R. Asada analyzed microbiological rock samples for mineral composition. Mineralogical properties were analyzed by a Rigaku RINT2200 X-ray diffractometer with $\mathrm{CuK \alpha}$ generated at 40 $\mathrm{kV}$ and $30 \mathrm{~mA}$. After air-drying the sample, the powders were pressed to fit the diffractometer sample holder. On the other hand, the XRD profiles of clay minerals were obtained from clay fractions deposited on glass slides in order. Furthermore, a fine mist of ethylene glycol (15\% in water) was sprayed on dry clay fractions for supplemental information about clay mineral identification.

\section{RESULTS}

Bulk mineralogical analyses were compiled for two sites drilled during Leg 193. These results are shown for the Snowcap diffuse venting area (Site 1188) and the Roman Ruins high-temperature area (Site 1189) in Figures F3 and F4, respectively. In addition, data for both sites are shown in Tables T1, T2, T3, and T4.

With the exception of fresh glassy rhyodacite and dacite near the seafloor, hydrothermal alteration is ubiquitous in the subsurface at Snowcap and Roman Ruins (Binns, Barriga, Miller, et al., 2002). Hydrothermal alteration is complex and multistage and includes pervasive re-
F1. Seafloor geology, p. 5.

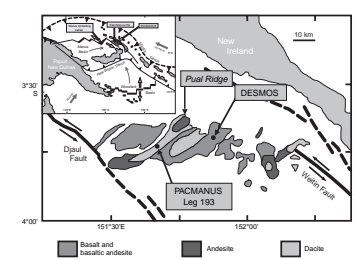

F2. Distribution of hydrothermal deposits, p. 6.

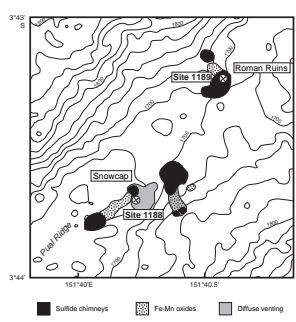

F3. Alteration, Site 1188, p. 7.

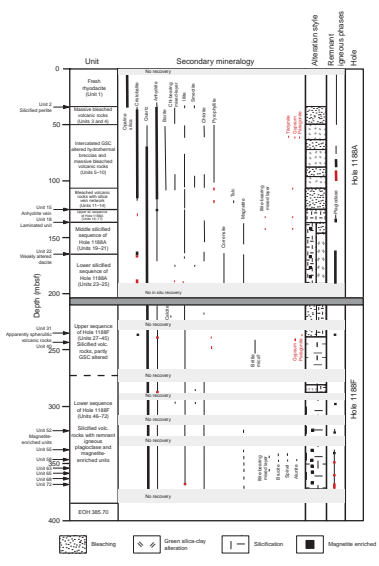

F4. Alteration, Holes 1189A and 1189B, p. 8.

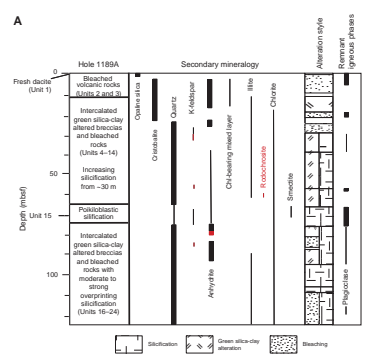

T1. Identified minerals, Hole $1188 \mathrm{~A}$, p. 10.

T2. Identified minerals, Hole 1188 F, p. 13. 
LACKSCHEWITZ ET AL.

placement of igneous material and alteration halos along anhydrite \pm pyrite \pm quartz veins. Mineral assemblages arising from hydrothermal alteration vary with depth and are complicated by overprinting relationships. At both sites, rocks are highly to completely altered to cristobalite and/or quartz, clay minerals (e.g., illite, chlorite, smectite, and mixed layers), anhydrite, and disseminated pyrite. In Hole 1188A a zone from $\sim 50$ to $120 \mathrm{mbsf}$ is characterized by a chlorite \pm illite-christobalite-plagioclase assemblage intercalated with a pyrophyllite assemblage (Fig. F3). A lower sequence below 120 mbsf in Hole 1189B comprises hydrothermal alteration features (Fig. F4B) that are distinctly different from those encountered at Snowcap (Site 1188). K-feldspar is abundant, and magnetite is rare. K-feldspar appears to be associated with quartz-chlorite alteration, which alternates with less strongly altered cristobalite-bearing rocks in the lower portion of Hole 1189B. A detailed description on clay mineral assemblages, clay and whole rock chemistry, and clay mineral isotopic compositions of the altered rocks from both sites is given in Lackschewitz et al. (2004).

\section{ACKNOWLEDGMENTS}

We thank R. Fonseca and F. Barriga for their helpful reviews. This research used samples and/or data provided by the Ocean Drilling Program (ODP). ODP is sponsored by the U.S. National Science Foundation (NSF) and participating countries under management of Joint Oceanographic Institutions (JOI), Inc. Funding for this research was provided by postcruise research grants to K. Lackschewitz and H. Paulick from the Deutsche Forschungsgemeinschaft.
T3. Identified minerals, Hole 1189A, p. 16.

T4. Identified minerals, Hole 1189B, p. 18. 


\section{REFERENCES}

Binns, R.A., Barriga, F.J.A.S., Miller, D.J., et al., 2002. Proc. ODP, Init. Repts., 193 [CDROM]. Available from: Ocean Drilling Program, Texas A\&M University, College Station TX 77845-9547, USA. [HTML]

Lackschewitz, K.S., Devey, C.W., Stoffers, P., Botz, R., Eisenhauer, A., Kummetz, M., Schmidt, M., and Singer, A., 2004. Mineralogical, geochemical and isotopic characteristics of hydrothermal alteration processes in the active, submarine, felsichosted PACMANUS field, Manus Basin, Papua New Guinea. Geochim. Cosmochim. Acta, 68(21):4405-4427. doi:10.1016/j.gca.2004.04.016

Shipboard Scientific Party, 2002. Leg 193 summary. In Binns, R.A., Barriga, F.J.A.S., Miller, D.J., et al., Proc. ODP, Init. Repts., 193: College Station TX (Ocean Drilling Program). [HTML] 
Figure F1. Seafloor geology of the eastern Manus Basin. East-northeast-trending neovolcanic edifices, extending between the active ends of the Djaul and Weitin transform faults, have yielded lavas ranging from picritic basalt to rhyodacite in composition (modified after Binns, Barriga, Miller, et al., 2002). Inset shows the regional tectonic setting of the PACMANUS hydrothermal site in the eastern Manus Basin. PACMANUS lies in the eastern Manus Rift zone, a pull-apart structure between two major transform faults (modified after Binns, Barriga, Miller, et al., 2002).

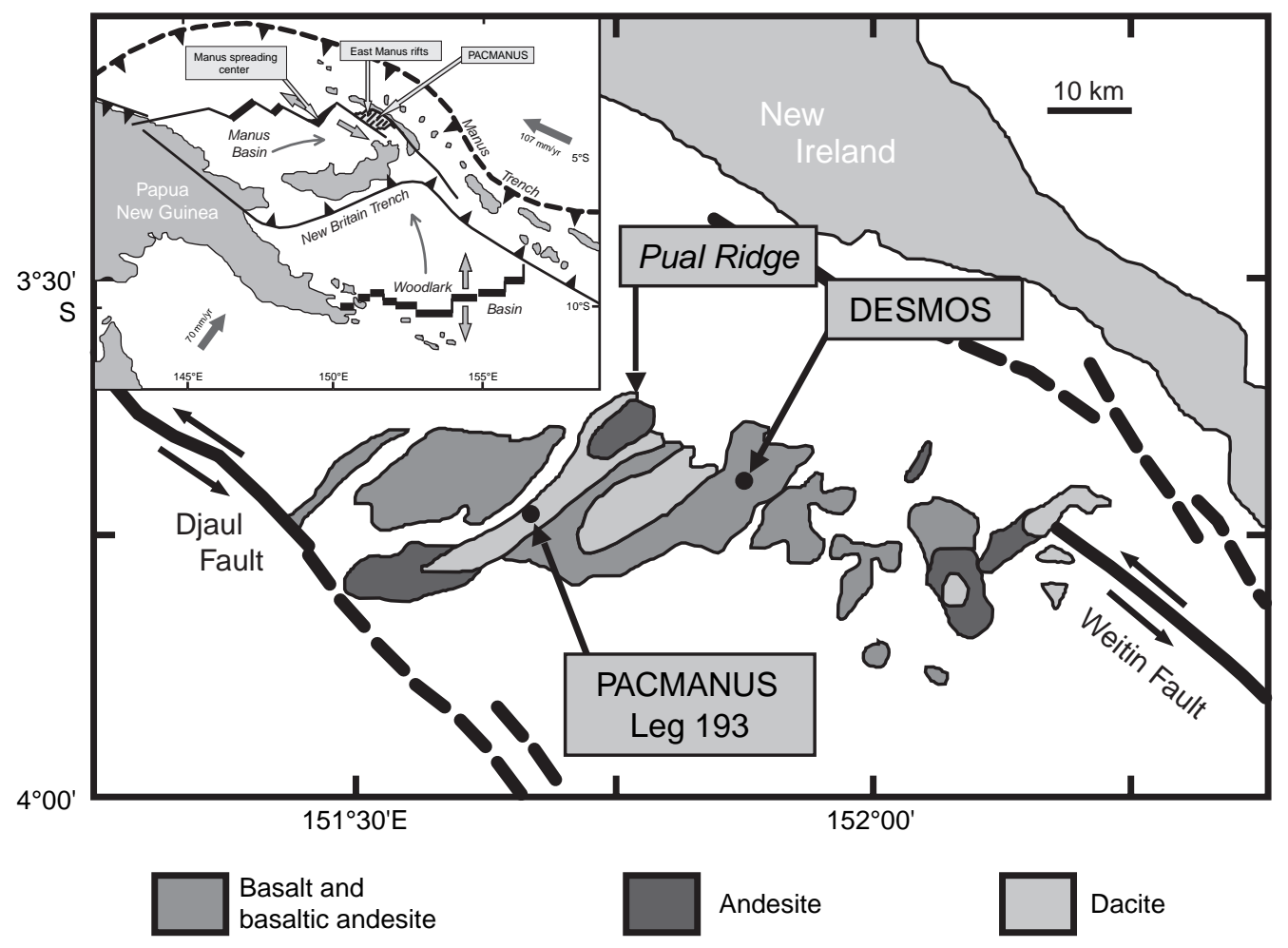


LACKSCHEWITZ ET AL.

Revised Alteration Phases

Figure F2. Distribution of hydrothermal deposits within the PACMANUS field along the crest of Pual Ridge and location of ODP Leg 193 drill hole sites. The map is based on bottom-tow photography and submersible dive observations from several PACMANUS cruises (modified after Binns, Barriga, Miller, et al., 2002).

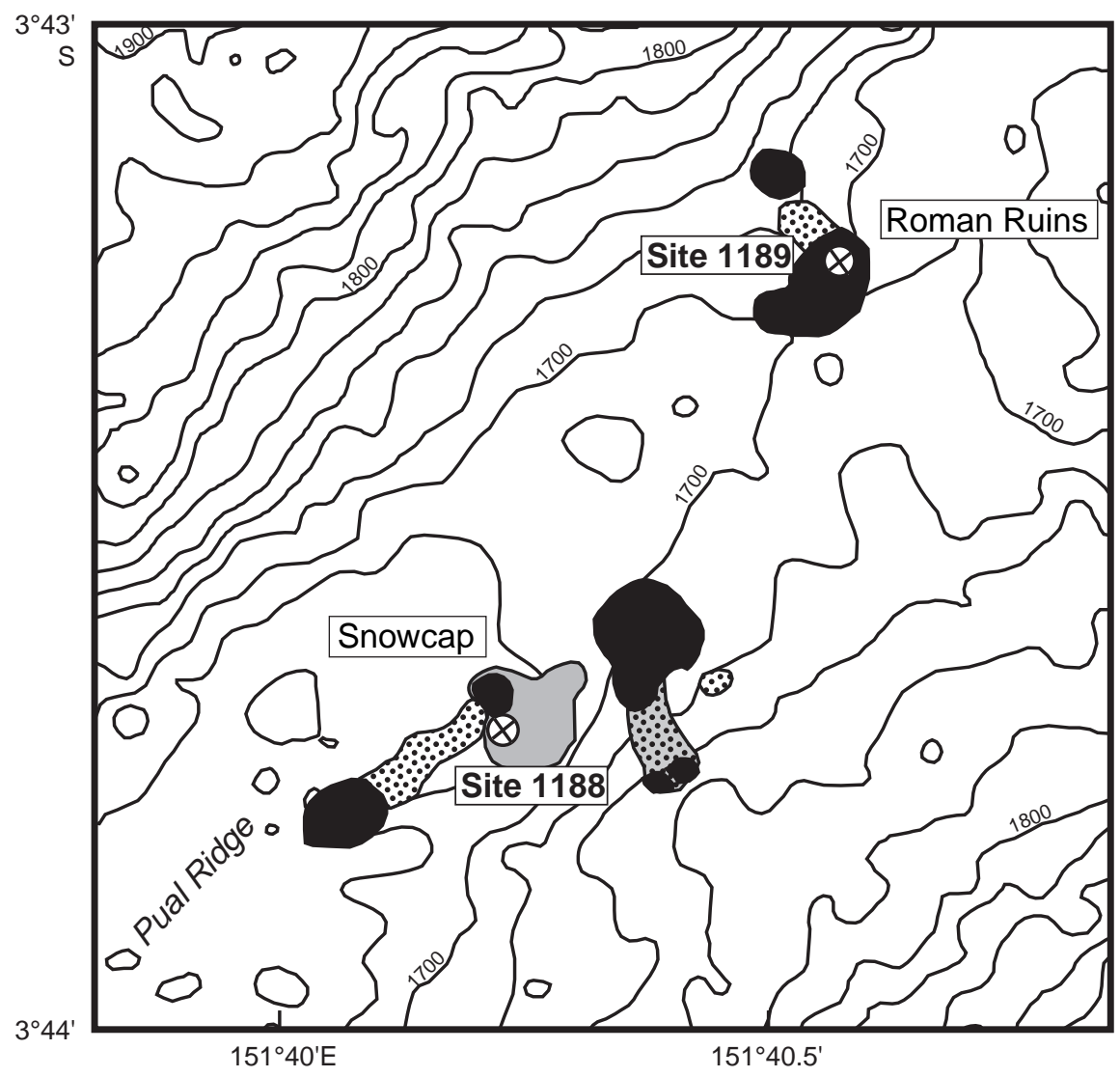


LACKSCHEWITZ ET AL.

Revised Alteration Phases

Figure F3. Summary of lithostratigraphic units, alteration style, and distribution of alteration phases at Site 1188, as a revision of fig. F16 in Shipboard Scientific Party (2002). Revised and newly obtained alteration phases are shown in red bars or lines. GSC = green silica clay, $\mathrm{EOH}=$ end of hole.

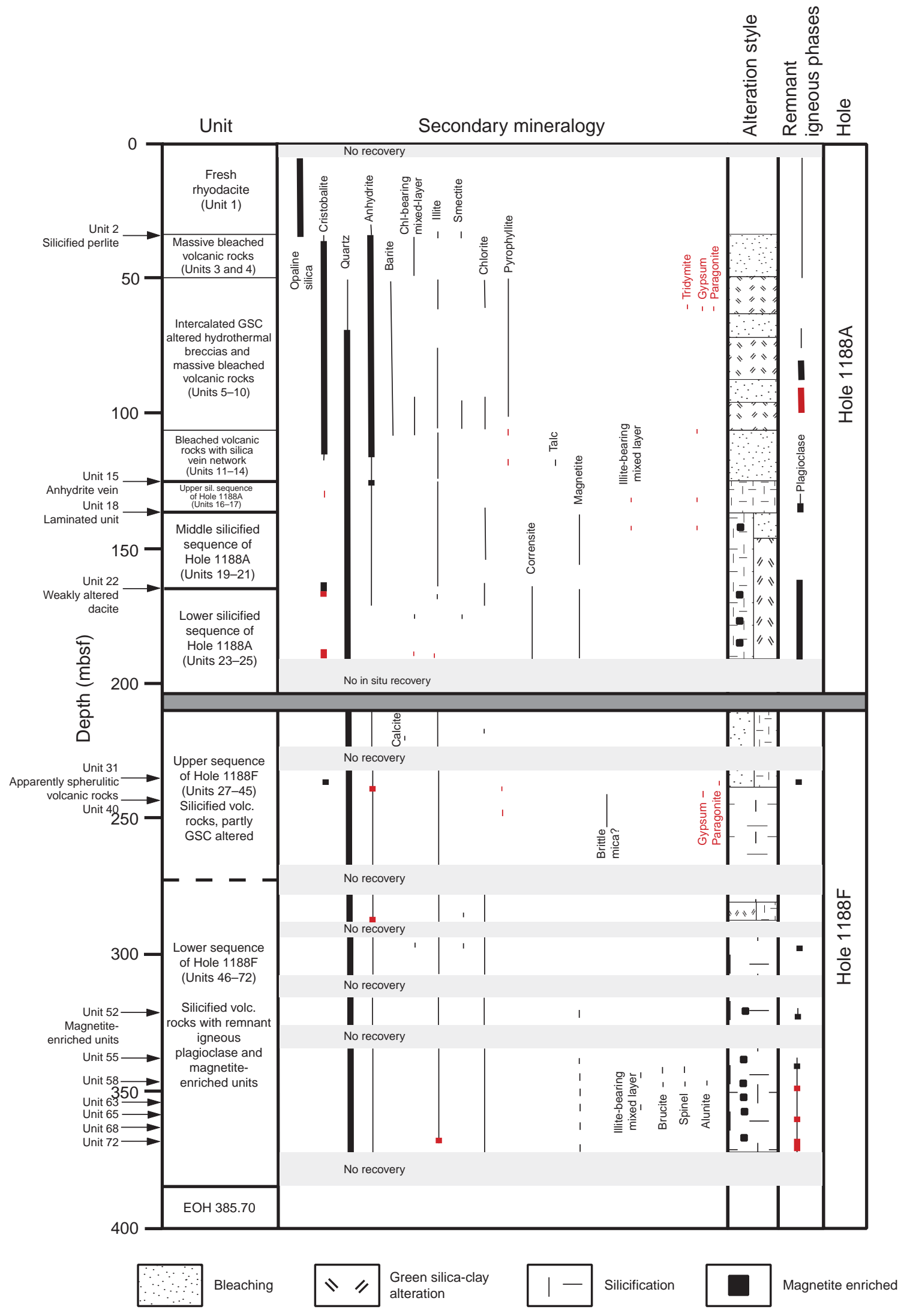


LACKSCHEWITZ ET AL.

Revised Alteration Phases

Figure F4. Summary of lithostratigraphic units, alteration style, and distribution of alteration phases as a revision of fig. F29 in Shipboard Scientific Party (2002). Revised and newly obtained alteration phases are shown in red bars or lines. A. Hole 1189A. (Continued on next page.)

A

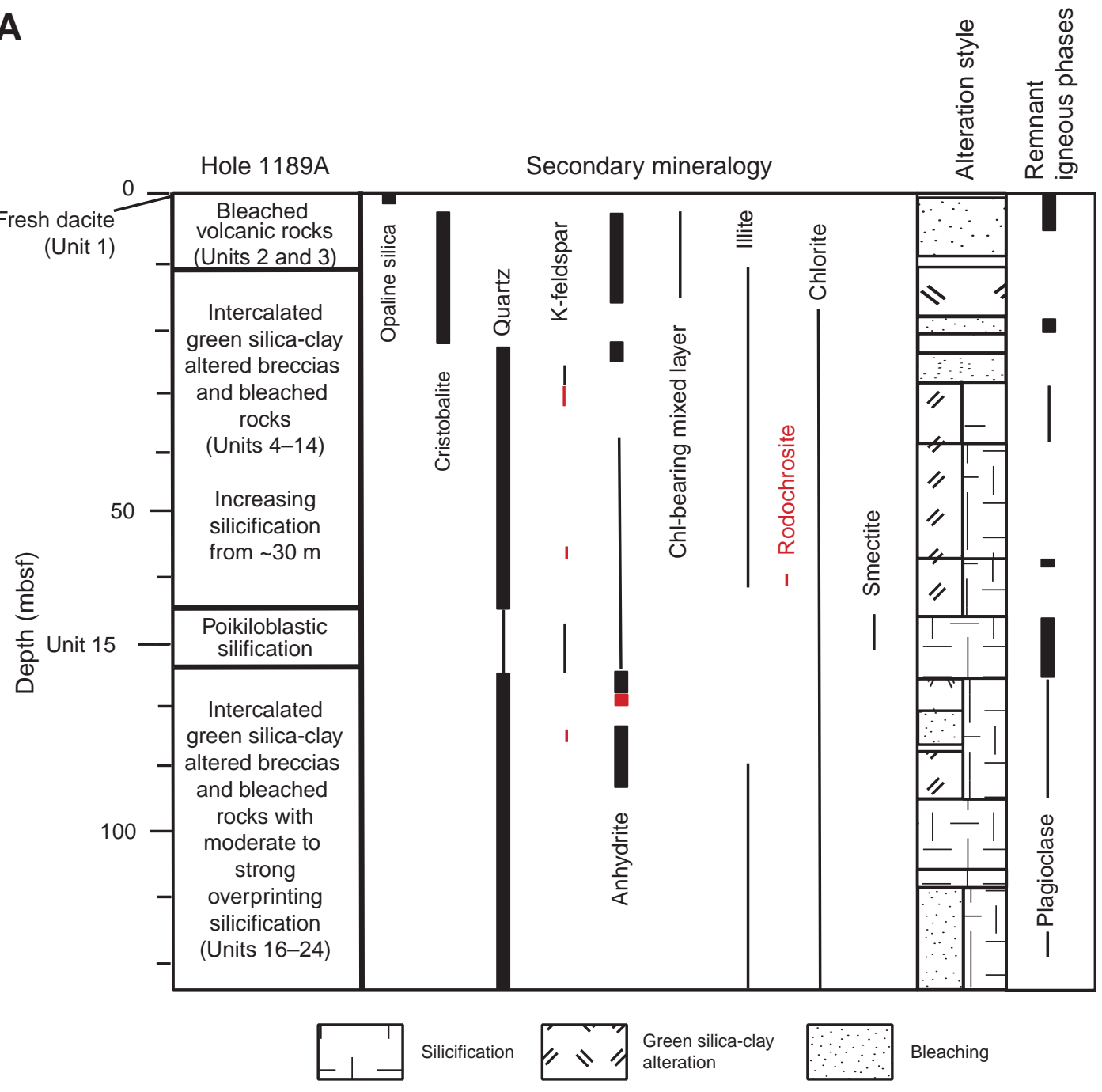


LACKSCHEWITZ ET AL.

Figure F4 (continued). B. Hole 1189B.

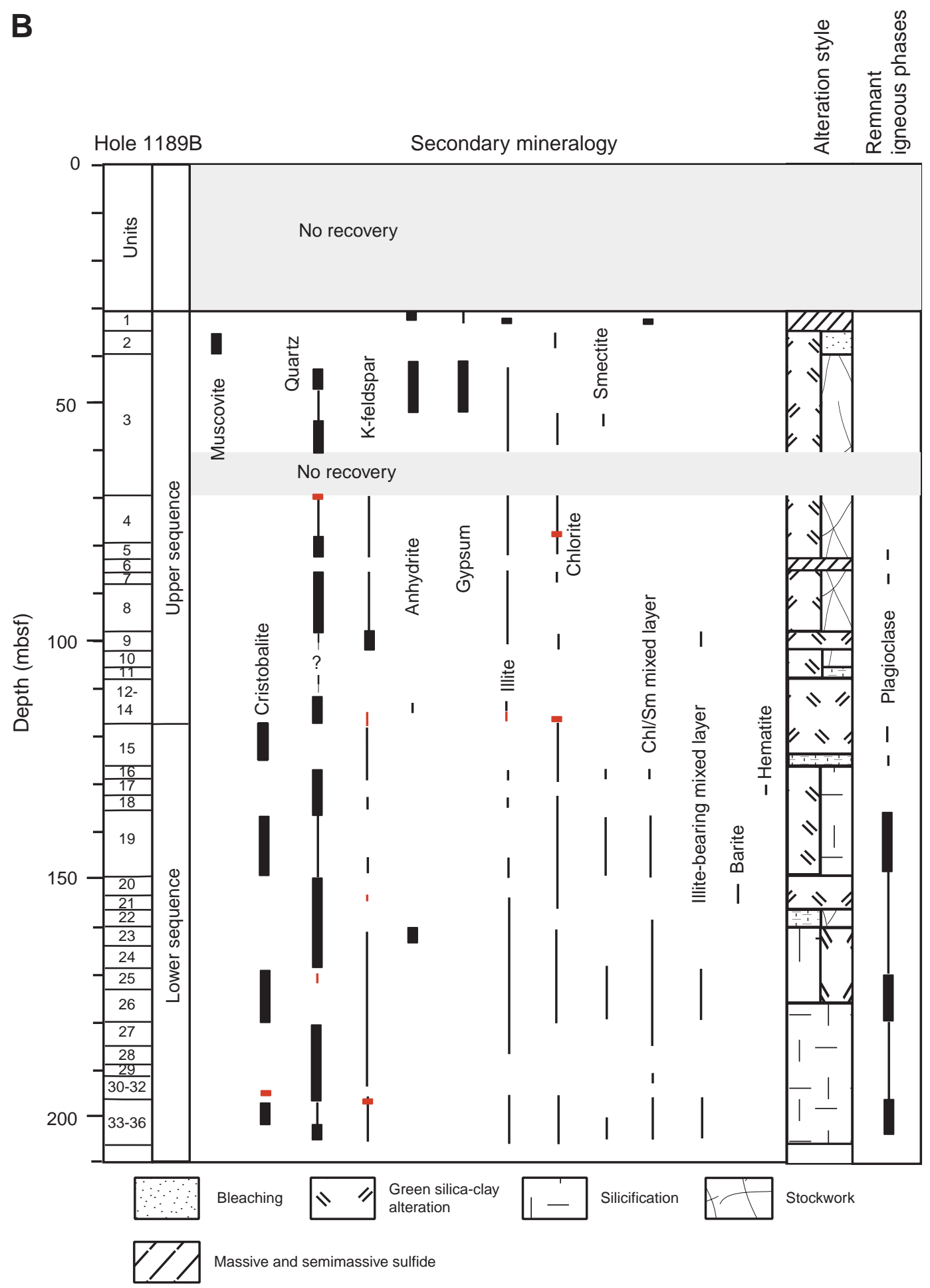


Table T1. Minerals identified in altered whole rocks by XRD analysis, Hole 1188A. (See table notes. Continued on next two pages.)

\begin{tabular}{|c|c|c|c|c|c|}
\hline $\begin{array}{l}\text { Core, section, } \\
\text { interval }(\mathrm{cm})\end{array}$ & $\begin{array}{l}\text { Depth } \\
\text { (mbsf) }\end{array}$ & Unit & Lab & Description & XRD identification \\
\hline \multicolumn{6}{|l|}{ 193-1188A- } \\
\hline $2 \mathrm{R}-1,9-12$ & 9.69 & 1 & SBD & Moderately vesicular rhyodacite & Opaline silica, plagioclase (augite, smectite) \\
\hline $5 R-1,17-21$ & 33.80 & 2 & RA & Sparsely plagioclase-phyric, moderately vesicular, rhyodacite & Opaline silica, plagioclase (cristobalite) \\
\hline $5 R-1,38-40$ & 33.97 & 2 & HP & Completely altered aphyric volcanic rock with perlitic texture; cores of perlite & Opaline silica/volcanic glass \\
\hline $5 R-1,38-40$ & 33.97 & 2 & $\mathrm{HP}$ & Completely altered aphyric volcanic rock with perlitic texture; margins of perlite & Opaline silica/volcanic glass \\
\hline $5 R-1,38-40$ & 33.97 & 2 & $\mathrm{HP}$ & $\begin{array}{l}\text { Completely altered aphyric volcanic rock with perlitic texture; pseudomatrix between } \\
\text { perlite kernels }\end{array}$ & Opaline silica/volcanic glass (mixed-layer clays) \\
\hline $5 R-1,38-40$ & 33.98 & 2 & SBD & Completely altered aphyric volcanic rock with perlitic texture & Opaline silica (illite, smectite, pyrite, plagioclase) \\
\hline $5 R-1,42-45$ & 34.02 & 2 & SBD & Completely altered aphyric volcanic rock with perlitic texture & Opaline silica (plagioclase, anorthite, sepiolite, K-feldspar, pyrite, smectite) \\
\hline $7 \mathrm{R}-1,55-58$ & 48.80 & 4 & RA & Completely altered, moderately vesicular aphyric ?dacite & Cristobalite, pyrite (quartz, gypsum, illite, pyrophyllite) \\
\hline $7 \mathrm{R}-1,62-64$ & 48.82 & 4 & SBD & Gray rock with anhydrite-pyrite veins & Cristobalite, anhydrite (pyrite, plagioclase) \\
\hline 7R-1, 92-93 & 49.12 & 4 & SBD & Flow-banded rock with anhydrite-pyrite veins & $\begin{array}{l}\text { Cristobalite (anhydrite, pyrite, plagioclase, smectite, chlorite-bearing mixed-layer, } \\
\text { hematite) }\end{array}$ \\
\hline 7R-1, 114-119 & 49.34 & 5 & $\mathrm{HP}$ & Domainal altered, aphyric volcanic rock; light gray perlitic(?) domain & Cristobalite (anhydrite, pyrite, illite, "chlorite, tridymite") \\
\hline 7R-1, 114-119 & 49.34 & 5 & HP & Domainal altered, aphyric volcanic rock; dark gray pseudomatrix & Cristobalite, anhydrite, pyrite (mixed-layer clay, "tridymite") \\
\hline 7R-1, 119-120 & 49.39 & 5 & SBD & Jigsaw breccia with textures replaced by anhydrite and chlorite & Anhydrite, cristobalite (pyrite, chlorite, illite) \\
\hline $7 \mathrm{R}-1,145-147$ & 49.65 & 5 & SBD & Rock with a pseudoclastic texture & Anhydrite (cristobalite, pyrite, pyrophyllite, quartz) \\
\hline $7 \mathrm{R}-2,39-41$ & 50.09 & 6 & SBD & Completely altered hydrofractured volcanic rock & Cristobalite (anhydrite, pyrite, chlorite, illite, barite) \\
\hline $7 \mathrm{R}-2,51-53$ & 50.21 & 6 & SBD & Completely altered hydrofractured volcanic rock & Anhydrite, cristobalite (pyrite, quartz, illite, barite) \\
\hline $8 \mathrm{R}-1,13-17$ & 58.03 & 6 & $\mathrm{HP}$ & Domainal altered, perlitic volcanic rock; white, remnant perlite & Cristobalite (tridymite, pyrite, mixed-layer clays, "anhydrite") \\
\hline $8 \mathrm{R}-1,13-17$ & 58.03 & 6 & HP & Domainal altered, perlitic volcanic rock; dark gray pseudomatrix & Cristobalite, pyrite, (tridymite, mixed-layer clays, "anhydrite") \\
\hline $8 \mathrm{R}-1,13-17$ & 58.03 & 6 & $\mathrm{HP}$ & Domainal altered, perlitic volcanic rock; bulk rock & Cristobalite (tridymite, pyrite, mixed-layer clays, "anhydrite") \\
\hline $8 \mathrm{R}-1,66-70$ & 58.56 & 6 & $\mathrm{HP}$ & Completely altered hydrofractured, flow-banded volcanic rock & Cristobalite (chlorite, "pyrite, illite, anatase) \\
\hline $8 \mathrm{R}-1,66-70$ & 58.58 & 6 & SBD & Completely altered hydrofractured volcanic rock & Cristobalite (anhydrite, pyrite, chlorite, illite, barite) \\
\hline $8 R-1,108-112$ & 58.98 & 6 & $\mathrm{KL}$ & Completely altered hydrofractured volcanic rock & Cristobalite (chlorite, smectite, anhydrite, pyrite) \\
\hline $8 \mathrm{R}-1,124-127$ & 59.14 & 6 & SBD & Completely altered hydrofractured volcanic rock & Cristobalite (anhydrite, pyrite, illite, chlorite, barite) \\
\hline $8 \mathrm{R}-1,147-148$ & 59.37 & 7 & SBD & Completely bleached vesicular volcanic rock with silification & Cristobalite, anhydrite (pyrite, quartz, illite, barite) \\
\hline $8 \mathrm{R}-2,18-20$ & 59.6 & 7 & RA & Pervasively bleached, vesicular volcanic rock & Cristobalite (gypsum, pyrite, illite, chlorite, mixed-layer clays) \\
\hline $9 \mathrm{R}-1,5-9$ & 67.65 & 7 & $\mathrm{KL}$ & Completely bleached vesicular volcanic rock with silification & Cristobalite, anhydrite, quartz (pyrophyllite, pyrite) \\
\hline $9 \mathrm{R}-1,13-14$ & 67.73 & 7 & SBD & Completely bleached vesicular volcanic rock with silification & Cristobalite, anhydrite, quartz (pyrophyllite, pyrite, barite) \\
\hline $9 \mathrm{R}-1,67-70$ & 68.27 & 8 & SBD & Completely altered hydrofractured volcanic rock & Cristobalite (pyrophyllite, pyrite, anhydrite, barite) \\
\hline $9 \mathrm{R}-1,130-135$ & 68.9 & 8 & HP & Completely altered hydrofractured volcanic rock & $\begin{array}{l}\text { Cristobalite, anhydrite (quartz, pyrite, pyrophyllite, paragonite, gypsum "halite, } \\
\text { anatase") }\end{array}$ \\
\hline $9 \mathrm{R}-1,137-140$ & 69.0 & 8 & RA & Pervasively bleached, vesicular volcanic rock & Cristobalite, anhydrite (gypsum, pyrophyllite, quartz) \\
\hline $9 \mathrm{R}-2,79-81$ & 69.89 & 8 & SBD & Completely altered hydrofractured volcanic rock & Cristobalite (anhydrite, quartz, pyrite, illite) \\
\hline $10 \mathrm{R}-1,35-37$ & 77.65 & 8 & SBD & Completely altered hydrofractured volcanic rock & Quartz, cristobalite (pyrophyllite, barite, pyrite) \\
\hline 10R-1, 39-43 & 77.69 & 8 & $\mathrm{KL}$ & Completely altered hydrofractured volcanic rock & Quartz (cristobalite, anhydrite, pyrophyllite) \\
\hline $11 \mathrm{R}-1,20-26$ & 87.1 & 9 & $\mathrm{HP}$ & Domainal altered, gray to white, massive volcanic rock; white margin & Cristobalite, quartz, anhydrite (tridymite, illite, gypsum, "?pyrophyllite") \\
\hline $11 \mathrm{R}-1,20-26$ & 87.1 & 9 & $\mathrm{HP}$ & Domainal altered, gray to white, massive volcanic rock; gray central part & $\begin{array}{l}\text { Cristobalite, plagioclase, pyrite (quartz, anhydrite, rutile, "chlorite?, mixed-layer } \\
\text { clays") }\end{array}$ \\
\hline $11 \mathrm{R}-1,35-38$ & 87.25 & 9 & SBD & Completely bleached vesicular volcanic rock with silification & Cristobalite (plagioclase, quartz, anhydrite, pyrite, chlorite) \\
\hline $11 \mathrm{R}-1,56-57$ & 87.46 & 9 & SBD & Completely bleached vesicular volcanic rock with silification & Cristobalite, plagioclase, quartz (pyrite, anhydrite, illite, barite) \\
\hline $11 \mathrm{R}-1,99-100$ & 87.9 & 9 & RA & Completely altered, bleached, sparsely vesicular volcanic rock & Cristobalite, pyrite (gypsum, anhydrite, quartz, illite) \\
\hline $12 \mathrm{R}-1,51-52$ & 97.11 & 10 & SBD & Completely altered hydrofractured perlitic and flow-banded rock & Quartz, cristobalite, anhydrite (pyrophyllite, pyrite) \\
\hline $12 \mathrm{R}-1,58-61$ & 97.18 & 10 & $\mathrm{KL}$ & Completely altered hydrofractured perlitic and flow-banded rock & Cristobalite, plagioclase (chlorite, illite, quartz, anhydrite) \\
\hline $12 \mathrm{R}-1,69-70$ & 97.29 & 10 & SBD & Completely altered hydrofractured perlitic and flow-banded rock & $\begin{array}{l}\text { Cristobalite (anhydrite, pyrite, illite, chlorite, chlorite-bearing mixed-layer, smectite, } \\
\text { barite) }\end{array}$ \\
\hline $12 \mathrm{R}-1,108-111$ & 97.68 & 10 & $\mathrm{KL}$ & Completely altered hydrofractured perlitic and flow-banded rock & Cristobalite, plagioclase (quartz, chlorite, illite, pyrite) \\
\hline $12 \mathrm{R}-1,117-118$ & 97.77 & 10 & SBD & Completely altered hydrofractured perlitic and flow-banded rock & Cristobalite, plagioclase (quartz, pyrite, anhydrite, barite) \\
\hline $12 \mathrm{R}-1,128-140$ & 97.9 & 10 & RA & Completely altered, bleached, sparsely vesicular volcanic rock & Cristobalite, plagioclase (quartz, pyrite, chlorite, illite) \\
\hline
\end{tabular}

Cristobalite (anhydrite, pyrite, plagioclase, smectite, chlorite-bearing mixed-layer, hematite)

(tridymite, pyrite, mixed-layer clays, "anhydrite")

(Cristobalite, pyrite, (tridymite, mixed-layer clays, "anhydrite")

Cristobalite (chlorite, "pyrite, illite, anatase)

Cristobalite (anhydrite, pyrite, chlorite, illite, barite)

Cristobalite, anhydrite (pyrite, quartz, illite, barite)

Cristobalite (gypsum, pyrite, ilite, chlorite, mixed-layer clays)

Cristobalite, anhydrite, quartz (pyrophyllite, pyrite)

Cristobalite, anhydrite, quartz (pyrophyllite, pyrite, barite)

ristobalite (pyrophyllite, pyrite, anhydrite, barite)

anatase")
Cristobalite, anhydrite (gypsum, pyrophyllite, quartz)

Cristobalite (anhydrite, quartz, pyrite, illite)

(cristobalite, (pyrophylite, barite, pyite)

Cristobalite, quartz, anhydrite (tridymite, illite, gypsum, "2pyrophyllite")

Cristobalite, plagioclase, pyrite (quartz, anhydrite, rutile, "chlorite?, mixed-laye clays")

Cristobalite, pyrite (gypsum, anhydrite, quartz, illite)

Quartz, cristobalite, anhydrite (pyrophyllite, pyrite)

Cristobalite, plagioclase (quartz, chlorite, illite, pyrite)

Cristobalite, plagioclase (quartz, pyrite, chlorite, illite) 
Table T1 (continued).

\begin{tabular}{|c|c|c|c|c|c|}
\hline $\begin{array}{l}\text { Core, section, } \\
\text { interval }(\mathrm{cm})\end{array}$ & $\begin{array}{l}\text { Depth } \\
\text { (mbsf) }\end{array}$ & Unit & Lab & Description & XRD identification \\
\hline $12 \mathrm{R}-2,20-24$ & 98.28 & 10 & $\mathrm{KL}$ & Completely altered hydrofractured perlitic and flow-banded rock & Cristobalite, plagioclase (quartz, chlorite, illite, pyrite) \\
\hline $12 \mathrm{R}-2,48-51$ & 98.55 & 10 & HP & Completely altered flow-banded rock; light gray layers & Cristobalite, plagioclase (quartz "tridymite, pyrite, chlorite") \\
\hline $12 \mathrm{R}-2,48-51$ & 98.55 & 10 & HP & Completely altered flow-banded rock; dark gray layers & Cristobalite, plagioclase (quartz "chlorite") \\
\hline $12 \mathrm{R}-2,48-51$ & 98.56 & 10 & SBD & Completely altered hydrofractured perlitic and flow-banded rock & Cristobalite, plagioclase (quartz, pyrite, anhydrite, barite, chlorite) \\
\hline $13 \mathrm{R}-1,9-12$ & 106.39 & 11 & SBD & Completely altered, pervasively bleached volcanic rock & $\begin{array}{l}\text { Cristobalite, plagioclase (quartz, pyrite, anhydrite, barite, chlorite-bearing mixed- } \\
\text { layer) }\end{array}$ \\
\hline $13 R-1,50-53$ & 106.8 & 11 & RA & Completely altered, bleached, sparsely vesicular volcanic rock & Quartz, anhydrite (gypsum, pyrite, illite, pyrophyllite) \\
\hline 14R-1, 4-10 & 116.04 & 12 & SBD & Completely altered volcaniclastic, granule to pebble breccia. & Quartz (illite, pyrite) \\
\hline $14 \mathrm{R}-1,44-45$ & 116.44 & 13 & SBD & Pervasively bleached hydrofractured volcanic rock & Quartz (cristobalite, anhydrite, pyrite, illite, talc) \\
\hline $14 \mathrm{R}-1,55-60$ & 116.55 & 13 & $\mathrm{KL}$ & Pervasively bleached hydrofractured volcanic rock & Quartz, anhydrite (pyrophyllite, gypsum) \\
\hline $14 \mathrm{R}-1,86-89$ & 116.86 & 13 & HP & Pervasively bleached hydrofractured volcanic rock; average rock & Anhydrite (quartz, pyrite, gypsum, illite?, pyrophyllite?, mixed-layer clays?) \\
\hline 14R-1, 86-89 & 116.86 & 13 & HP & Pervasively bleached hydrofractured volcanic rock; white pseudoclastic domains & Quartz (anhydrite, anatase, paragonite?, pyrophyllite?, "pyrite, halite”) \\
\hline $14 \mathrm{R}-1,92-93$ & 116.92 & 13 & SBD & Pervasively bleached hydrofractured volcanic rock & Quartz, anhydrite (pyrite, illite, talc) \\
\hline $14 R-1,105-108$ & 117.27 & 14 & HP & Completely altered volcaniclastic breccia; cream-colored clast & Quartz, anhydrite (gypsum, paragonite?, mixed-layer clays? "tridymite, halite") \\
\hline $14 \mathrm{R}-1,105-108$ & 117.27 & 14 & HP & Completely altered volcaniclastic breccia; bulk rock & $\begin{array}{l}\text { Quartz, anhydrite (gypsum, paragonite?, mixed-layer clays?, "illite?, tridymite, } \\
\text { halite") }\end{array}$ \\
\hline $14 R-1,105-108$ & 117.29 & 14 & SBD & Completely altered volcaniclastic breccia & Quartz (anhydrite, illite) \\
\hline $15 R-1,14-21$ & 125.84 & 15 & SBD & Anhydrite vein & Anhydrite \\
\hline $15 R-1,56-59$ & 126.26 & 16 & $\mathrm{KL}$ & Completely bleached and silicified, vesicular volcanic rock & Quartz, plagioclase (cristobalite, anhydrite, chlorite, illite-bearing mixed-layer) \\
\hline $15 R-1,76-80$ & 126.46 & 16 & SBD & Completely bleached and silicified, vesicular volcanic rock & Quartz (illite, pyrite, anhydrite) \\
\hline $15 R-1,80-81$ & 126.50 & 16 & RA & Crustiform anhydrite-pyrite vein & $\begin{array}{l}\text { Quartz, pyrite, plagioclase (gypsum, anhydrite, illite, chlorite, mixed-layer clays, } \\
\text { cristobalite) }\end{array}$ \\
\hline 16R-1, 77-78 & 136.17 & 17 & SBD & Completely silicified, sparsely vesicular volcanic rock & Quartz (anhydrite, plagioclase, illite, pyrite, chlorite) \\
\hline 16R-1, 95-98 & 136.35 & 17 & SBD & Completely silicified, sparsely vesicular volcanic rock & Quartz (anhydrite, plagioclase, illite, chlorite) \\
\hline 16R-1, 139-143 & 136.79 & 18 & HP & Massive gray volcanic rock with anhydrite-vein; white vein & Quartz, anhydrite (illite "chlorite, gypsum, rutile") \\
\hline 16R-1, 139-143 & 136.79 & 18 & HP & Massive gray volcanic rock with anhydrite-vein; gray groundmass & Quartz, plagioclase (pyrite "chlorite") \\
\hline 16R-2, 12-15 & 136.98 & 18 & SBD & Gray-green finely laminated, strongly silicified volcanic rock & Quartz, plagioclase (pyrite, chlorite, anhydrite) \\
\hline $16 \mathrm{R}-2,40-43$ & 137.26 & 18 & SBD & Gray-green finely laminated, strongly silicified volcanic rock & Quartz, plagioclase (pyrite, chlorite, anhydrite) \\
\hline $16 \mathrm{R}-2,51-52$ & 137.37 & 18 & SBD & Gray-green finely laminated, strongly silicified volcanic rock & Quartz, plagioclase (chlorite, anhydrite, pyrite) \\
\hline 16R-2, 68-69 & 137.54 & 18 & SBD & Gray-green finely laminated, strongly silicified volcanic rock & Quartz, plagioclase (chlorite, pyrite, anhydrite) \\
\hline 17R-1, 24-28 & 145.34 & 19 & HP & Silicified, bleached volcanic rock & Quartz, anhydrite (pyrite, illite?, mixed-layer clays, gypsum "chlorite") \\
\hline 17R-1, 38-41 & 145.48 & 19 & $\mathrm{KL}$ & Silicified, bleached volcanic rock & Quartz (anhydrite, pyrite, chlorite, illite-bearing mixed-layer) \\
\hline 17R-1, 90-93 & 146.00 & 19 & SBD & Silicified, bleached volcanic rock & Quartz (illite, pyrite, anhydrite, chlorite) \\
\hline $17 R-1,123-124$ & 146.33 & 19 & SBD & Silicified, bleached volcanic rock & Quartz (anhydrite, illite) \\
\hline $18 \mathrm{R}-1,28-29$ & 154.98 & 19 & SBD & Silicified, bleached volcanic rock & Quartz, anhydrite (magnetite, illite) \\
\hline 18R-1, 87-97 & 155.57 & 20 & SBD & Silicified and bleached moderately vesicular volcanic rock & Quartz (anhydrite) \\
\hline 19R-1, 41-46 & 164.71 & 21 & HP & Silicified volcanic rock & Quartz (cristobalite, plagioclase, chlorite?, mixed-layer clays? "tridymite") \\
\hline $19 \mathrm{R}-1,51-57$ & 164.81 & 21 & SBD & Silicified volcanic rock & Cristobalite, plagioclase (quartz, chlorite) \\
\hline 19R-1, 73-76 & 165.03 & 22 & SBD & Moderately altered, vesicular volcanic rock & Cristobalite, plagioclase (chlorite) \\
\hline $19 \mathrm{R}-1,86-90$ & 165.16 & 22 & HP & Moderately altered, vesicular volcanic rock & Cristobalite, plagioclase ("anhydrite, chlorite") \\
\hline $20 \mathrm{R}-1,8-12$ & 173.98 & 23 & $\mathrm{KL}$ & Silicified, moderately chloritic magnetite-bearing volcanic rock & Cristobalite, quartz, plagioclase, chlorite-bearing mixed-layer (anhydrite, pyrite) \\
\hline 20R-1, 46-47 & 174.36 & 23 & SBD & Silicified, moderately chloritic magnetite-bearing volcanic rock & Quartz (plagioclase, anhydrite, corrensite, magnetite, illite, pyrite) \\
\hline 20R-1, 68-72 & 174.58 & 23 & $\mathrm{KL}$ & Silicified, moderately chloritic magnetite-bearing volcanic rock & Quartz, plagioclase (chlorite, pyrite) \\
\hline $20 \mathrm{R}-1,75-76$ & 174.65 & 23 & SBD & Silicified, moderately chloritic magnetite-bearing volcanic rock & Quartz, plagioclase (magnetite, pyrite, chlorite) \\
\hline 20R-1, 92-95 & 174.82 & 24 & SBD & Distinctive dark green silicified rock & Quartz, plagioclase (magnetite, pyrite, smectite, anhydrite, mixed-layers) \\
\hline 20R-1, 95-102 & 174.85 & 24 & HP & Distinctive dark green silicified rock; abundant white dots & Quartz (plagioclase, pyrite) \\
\hline 20R-1, 95-102 & 174.85 & 24 & HP & Distinctive dark green silicified rock; minor white dots & Quartz, plagioclase (pyrite "anhydrite, mixed-layer clays") \\
\hline 20R-1, 102-103 & 174.92 & 24 & SBD & Distinctive dark green silicified rock & Quartz, plagioclase (magnetite, pyrite, corrensite, anhydrite) \\
\hline $21 \mathrm{R}-1,20-21$ & 183.30 & 25 & SBD & Green-black rock with green clay in a silica-pyrite stockwork & Quartz (plagioclase, magnetite, corrensite, pyrite) \\
\hline $21 \mathrm{R}-1,43-47$ & 183.53 & 25 & $\mathrm{KL}$ & Green-black rock with green clay in a silica-pyrite stockwork & Quartz, plagioclase (magnetite, chlorite-bearing mixed-layer) \\
\hline
\end{tabular}


Table T1 (continued).

\begin{tabular}{|c|c|c|c|c|c|}
\hline $\begin{array}{l}\text { Core, section, } \\
\text { interval }(\mathrm{cm})\end{array}$ & $\begin{array}{l}\text { Depth } \\
\text { (mbsf) }\end{array}$ & Unit & Lab & Description & XRD identification \\
\hline $21 \mathrm{R}-1,77-81$ & 183.87 & 25 & $\mathrm{KL}$ & Green-black rock with green clay in a silica-pyrite stockwork & $\begin{array}{l}\text { Cristobalite, plagioclase (quartz, magnetite, chlorite-bearing mixed-layer, pyrite, } \\
\text { anhydrite) }\end{array}$ \\
\hline 21R-1, 105-114 & 184.2 & 25 & RA & Patchy quartz, magnetite, chlorite-clay bearing rock. & Quartz, plagioclase (cristobalite, pyrite, chlorite, illite, gypsum) \\
\hline $21 \mathrm{R}-1,142-146$ & 184.52 & 25 & $\mathrm{KL}$ & Green-black rock with green clay in a silica-pyrite stockwork & Cristobalite, plagioclase (quartz, chlorite-bearing mixed-layer, illite, pyrite, anhydrite) \\
\hline
\end{tabular}


Table T2. Minerals identified in altered whole rocks by XRD analysis, Hole 1188F. (See table notes. Continued on next two pages.)

\begin{tabular}{|c|c|c|c|c|c|}
\hline $\begin{array}{l}\text { Core, section, } \\
\text { interval }(\mathrm{cm})\end{array}$ & $\begin{array}{l}\text { Depth } \\
\text { (mbsf) }\end{array}$ & Unit & Lab & Description & XRD identification \\
\hline \multicolumn{6}{|l|}{$193-1188 \mathrm{~F}-$} \\
\hline $1 Z-1,4-5$ & 218.04 & 27 & SBD & Completely altered massive rock & Quartz (anhydrite, illite, "pyrite") \\
\hline $1 Z-1,29-30$ & 218.29 & 27 & SBD & Completely altered massive rock & Quartz (anhydrite, illite, pyrite) \\
\hline $1 Z-1,87-88$ & 218.87 & 27 & SBD & Completely altered massive rock & Quartz (anhydrite, illite, pyrite) \\
\hline $1 Z-2,4-5$ & 219.24 & 27 & SBD & Completely altered massive rock & Quartz (anhydrite, illite) \\
\hline $1 Z-2,37-38$ & 219.57 & 27 & SBD & Completely altered massive rock & Quartz (anhydrite, illite, "pyrite") \\
\hline $1 Z-3,20-21$ & 220.60 & 27 & SBD & Completely altered massive rock & Quartz (anhydrite, illite) \\
\hline $1 Z-3,34-35$ & 220.74 & 27 & SBD & Completely altered massive rock & Quartz (anhydrite, illite) \\
\hline $1 Z-3,35-36$ & 220.75 & 27 & SBD & Completely altered massive rock (including anhydrite vein) & Quartz (anhydrite, illite) \\
\hline $1 Z-3,85-86$ & 221.25 & 28 & SBD & Completely altered, silicified, and green clay-bearing massive rock & Quartz (chlorite, illite, anhydrite, pyrite) \\
\hline $1 Z-3,106-108$ & 221.46 & 28 & SBD & Completely altered, silicified, and green clay-bearing massive rock & Quartz (anhydrite, illite, pyrite, "chlorite") \\
\hline $1 Z-4,63-65$ & 222.2 & 28 & RA & Completely altered, silicified aphyric volcanic rock & Quartz, anhydrite, pyrite (gypsum, illite) \\
\hline $1 Z-4,90-91$ & 222.50 & 28 & SBD & Completely altered, silicified, and green clay-bearing massive rock & Quartz (anhydrite, illite, "pyrite") \\
\hline $2 Z-1,44-45$ & 221.94 & 29 & SBD & Completely altered, silicified aphyric massive rock & Quartz (anhydrite, illite, pyrite) \\
\hline $2 Z-1,73-74$ & 222.23 & 29 & SBD & Completely altered, silicified aphyric massive rock & Quartz, calcite (anhydrite, illite, "kaolinite?") \\
\hline $3 Z-1,6-7$ & 222.66 & 29 & SBD & Completely altered, silicified aphyric massive rock & Quartz (illite, anhydrite, "pyrite") \\
\hline $3 Z-2,40-41$ & 224.45 & 30 & SBD & Completely altered, brecciated, clay-rich rock & Quartz (anhydrite, illite, pyrite) \\
\hline $3 Z-2,70-75$ & 224.8 & 30 & RA & Completely altered, brecciated, volcanic rock & Quartz (pyrite, chlorite, illite, mixed-layer clays, "anhydrite") \\
\hline $3 Z-2,144-145$ & 225.49 & 30 & SBD & Completely altered, brecciated, clay-rich rock & Quartz (anhydrite, chlorite, illite) \\
\hline $3 Z-2,150-151$ & 225.55 & 30 & SBD & Completely altered, brecciated, clay-rich rock & Quartz (anhydrite, illite, pyrite) \\
\hline $6 Z-1,20-21$ & 233.30 & 30 & SBD & Completely altered, brecciated, clay-rich rock & Quartz (anhydrite, illite, chlorite, pyrite) \\
\hline $6 Z-1,46-47$ & 233.56 & 31 & $\mathrm{HP}$ & Highly altered, spheroidal volcanic rock; spheroid-separate & Cristobalite, plagioclase (chlorite "quartz") \\
\hline $6 Z-1,46-47$ & 233.56 & 31 & HP & Highly altered volcanic rock; groundmass separate & Quartz, cristobalite (chlorite, pyrite "anhydrite, plagioclase") \\
\hline $6 Z-1,46-47$ & 233.56 & 31 & SBD & Highly altered volcanic rock & Cristobalite, plagioclase (chlorite, pyrite) \\
\hline $6 Z-1,77-78$ & 233.87 & 32 & SBD & Completely altered, silicified massive aphyric rock & Quartz (anhydrite, illite) \\
\hline $6 Z-2,47-48$ & 234.81 & 33 & SBD & Silicified volcanic fragments embedded in soft gray clay & Quartz (illite, pyrite, anhydrite) \\
\hline $7 Z-1,41-45$ & 235.41 & 34 & SBD & Silicified massive aphyric rock & Quartz (anhydrite, illite, "pyrite") \\
\hline 7Z-1, 104-107 & 236.04 & 35 & SBD & Silicified, plagioclase-phyric rock & Quartz (anhydrite, illite) \\
\hline $7 Z-2,3-6$ & 236.51 & 35 & SBD & Silicified, plagioclase-phyric rock & Quartz (anhydrite, illite, "pyrite") \\
\hline $8 Z-1,16-20$ & 236.40 & 36 & RA & Completely altered, silicified, massive, aphyric volcanic rock & Quartz, anhydrite, pyrite (illite, pyrophyllite) \\
\hline $8 Z-1,26$ & 236.46 & 36 & HP & Silicified massive aphyric rock; light green, amygdaloidal central part & Quartz (anhydrite, pyrite "chlorite, illite?") \\
\hline $8 Z-1,26$ & 236.46 & 36 & HP & Silicified massive aphyric rock; white, alteration halo (piece margin) & $\begin{array}{l}\text { Quartz, anhydrite (paragonite, mixed-layer clays, pyrophyllite?, illite? "rutile, } \\
\text { gypsum") }\end{array}$ \\
\hline $8 Z-1,68-71$ & 236.88 & 36 & SBD & Silicified massive aphyric rock & Quartz (anhydrite, illite) \\
\hline $9 Z-1,9-12$ & 237.80 & 37 & $\mathrm{HP}$ & Silicified clastic rock; white clasts & Anhydrite (quartz, illite, paragonite "gypsum, halite") \\
\hline $9 Z-1,9-12$ & 237.80 & 37 & HP & Silicified clastic rock; gray groundmass & Quartz, illite (anhydrite) \\
\hline $9 Z-1,9-12$ & 237.79 & 37 & SBD & Silicified clastic rock & Quartz (anhydrite, illite) \\
\hline $11 \mathrm{G}-1,53-56$ & 239.43 & 38 & SBD & Silicified massive aphyric rock & Quartz (anhydrite, illite, "pyrite") \\
\hline $11 \mathrm{G}-1,138-141$ & 240.28 & 39 & SBD & Silicified plagioclase-phyric, slightly vesicular volcanic rock & Quartz (anhydrite, pyrite, illite) \\
\hline $13 Z-1,0-23$ & 241.40 & 41 & $\mathrm{HP}$ & Silicified plagioclase-phyric volcanic rock; white alteration halo (margin) & Anhydrite, quartz (pyrite, illite "halite") \\
\hline $13 Z-1,0-23$ & 241.40 & 41 & HP & Silicified plagioclase-phyric volcanic rock; gray central part & Quartz (pyrite, illite "anhydrite") \\
\hline 13Z-1, 74-78 & 242.1 & 41 & RA & Silicified, sparsely plagioclase-phyric, sparsely vesicular rock & Quartz, anhydrite, gypsum, pyrite (plagioclase, illite, pyrophyllite) \\
\hline $13 Z-1,118-121$ & 242.58 & 41 & SBD & Silicified plagioclase-phyric volcanic rock & Quartz, anhydrite, pyrite, illite) \\
\hline $13 Z-2,51-55$ & 243.27 & 41 & SBD & Silicified plagioclase-phyric volcanic rock & Quartz (anhydrite, illite, “pyrite) \\
\hline $13 Z-2,55-70$ & 243.31 & 41 & SBD & Silicified plagioclase-phyric volcanic rock & Quartz (anhydrite, pyrite, illite, "chlorite") \\
\hline $14 Z-1,134-139$ & 247.24 & 41 & SBD & Silicified plagioclase-phyric volcanic rock & Quartz (anhydrite, brittle mica?, pyrite) \\
\hline $15 Z-1,66-69$ & 251.06 & 42 & SBD & Silicified, aphyric rock & Quartz (anhydrite, brittle mica?, pyrite) \\
\hline $15 Z-1,90-95$ & 251.30 & 42 & SBD & Silicified, aphyric rock & Quartz (anhydrite, illite, brittle mica?, pyrite) \\
\hline $16 Z-1,41-43$ & 255.31 & 44 & SBD & Silicified, brecciated, flow-banded, aphyric rock & Quartz (illite, pyrite, "anhydrite") \\
\hline
\end{tabular}


Table T2 (continued).

\begin{tabular}{|c|c|c|c|c|c|}
\hline $\begin{array}{l}\text { Core, section, } \\
\text { interval }(\mathrm{cm})\end{array}$ & $\begin{array}{l}\text { Depth } \\
\text { (mbsf) }\end{array}$ & Unit & Lab & Description & XRD identification \\
\hline $16 Z-1,88-90$ & 255.8 & 44 & RA & Completely altered, silicified, sparsely plagioclase-phyric volcanic rock & Quartz, pyrite (illite, pyrophyllite) \\
\hline $16 Z-1,139-141$ & 256.29 & 44 & SBD & Silicified, brecciated, flow-banded, aphyric rock & Quartz (pyrite, illite, brittle mica?, "anhydrite") \\
\hline $19 Z-1,0-41$ & 268.67 & 45 & HP & Silicified plagioclase-phyric volcanic rock & Quartz (anhydrite, pyrite, illite?, mixed-layer clays?) \\
\hline $22 Z-1,63-67$ & 282.73 & 46 & SBD & Completely altered, silicified, aphyric, volcanic rock & Quartz (anhydrite, pyrite, illite, chlorite) \\
\hline $22 Z-1,85-87$ & 283.0 & 46 & RA & Completely altered, silicified, aphyric, massive volcanic rock & Quartz, pyrite (gypsum, plagioclase, chlorite, illite, mixed-layer clays, "pyrophyllite") \\
\hline $23 Z-1,25-26$ & 286.85 & 48 & SBD & White halo of Piece 3 & Quartz (anhydrite, illite, pyrite, chlorite) \\
\hline $23 Z-1,26-27$ & 286.86 & 48 & SBD & Gray kernel of Piece 3 & Quartz (chlorite, illite, pyrite, "anhydrite, smectite") \\
\hline $23 Z-1,102-103$ & 287.62 & 49 & SBD & Silicified, green clay-bearing aphyric volcanic rock & Quartz (anhydrite, chlorite, illite, "pyrite") \\
\hline $23 Z-2,13-14$ & 288.23 & 49 & SBD & White crust of Piece 2 (anhydrite vein) & Anhydrite ("quartz") \\
\hline $23 Z-2,24-25$ & 288.34 & 49 & SBD & Silicified, green clay-bearing aphyric volcanic rock & Quartz (anhydrite, chlorite, illite, pyrite) \\
\hline $23 Z-2,45-46$ & 288.55 & 49 & SBD & Silicified, green clay-bearing aphyric volcanic rock & Quartz (anhydrite, chlorite, illite, "pyrite") \\
\hline $23 Z-2,56-63$ & 288.66 & 49 & HP & Silicified, green clay-bearing aphyric volcanic rock; light green domain & Quartz, chlorite (anhydrite, illite "pyrite, rutile, gypsum") \\
\hline $23 Z-2,56-63$ & 288.66 & 49 & HP & Silicified, green clay-bearing aphyric volcanic rock; light yellow domain & Quartz, anhydrite (pyrite, illite "rutile") \\
\hline $25 Z-1,24-26$ & 295.84 & 49 & SBD & Silicified, green clay-bearing aphyric volcanic rock & Quartz, plagioclase (chlorite, pyrite, "anhydrite") \\
\hline $26 Z-1,20-23$ & 300.30 & 49 & SBD & Silicified, green clay-bearing aphyric volcanic rock & Quartz (anhydrite, pyrite, illite, chlorite, chlorite-bearing mixed-layer, "smectite") \\
\hline $26 Z-2,34-36$ & 301.5 & 50 & RA & Completely altered, silicified, sparsely plagioclase-phyric rock & Quartz, anhydrite, pyrite (gypsum, chlorite, illite) \\
\hline $26 Z-2,60-61$ & 301.78 & 50 & SBD & Silicified, plagioclase-phyric volcanic rock & Quartz (chlorite, illite, anhydrite, pyrite) \\
\hline $27 Z-1,22-23$ & 304.82 & 50 & SBD & Silicified, plagioclase-phyric volcanic rock & Quartz (anhydrite, pyrite, plagioclase, illite, chlorite) \\
\hline $30 Z-1,5-7$ & 318.15 & 51 & SBD & Completely altered, variably silicified breccia & Quartz (plagioclase, illite, chlorite, "pyrite") \\
\hline $30 Z-1,13-20$ & 318.23 & 51 & HP & Completely altered, variably silicified, magnetite-bearing, breccia & Quartz (plagioclase, illite "chlorite, magnetite") \\
\hline $31 Z-1,1-3$ & 322.61 & 52 & SBD & Silicified, slightly plagioclase-magnetite phyric volcanic rock & Quartz, plagioclase (chlorite, magnetite, pyrite, "anhydrite") \\
\hline $31 Z-1,39-42$ & 322.99 & 53 & SBD & Completely altered, variably silicified breccia & Quartz (pyrite, illite, chlorite, "anhydrite") \\
\hline $34 Z-1,22-23$ & 336.62 & 54 & SBD & Completely altered aphyric volcanic rock & Quartz (anhydrite, illite, pyrite, "plagioclase") \\
\hline $34 Z-1,27-28$ & 336.67 & 55 & SBD & $\begin{array}{l}\text { Completely altered volcanic rock and breccia with variable black, late-stage } \\
\text { magnetite-bearing alteration }\end{array}$ & Magnetite (illite, quartz, plagioclase, chlorite, pyrite, Fe-spinel, "brucite") \\
\hline
\end{tabular}

34Z-1, 40-41 $336.80 \quad 55 \quad$ SBD $\quad$ Completely altered volcanic rock and breccia with variable black, late-stage magnetite-bearing alteration

34Z-1, 89-90 $\quad 337.29 \quad 56 \quad$ SBD Completely altered and variably silicified volcanic rock breccia

34Z-1, 122-130 $337.63 \quad 56 \quad$ HP $\quad$ Completely altered and variably silicified volcanic rock breccia

$\begin{array}{lllll}35 Z-1,5-6 & 340.05 & 57 & \text { SBD } & \text { Silicified, sparsely vesicular, aphyric, massive volcanic rock }\end{array}$

$\begin{array}{lllll}35 Z-1,46-48 & 340.46 & 57 & \text { SBD } & \text { Silicified, sparsely vesicular, aphyric, massive volcanic rock }\end{array}$

35Z-1, 140-141 $341.40 \quad 57 \quad$ SBD $\quad$ Silicified, sparsely vesicular, aphyric, massive volcanic rock

$\begin{array}{lllll}35 Z-2,48-49 & 341.98 & 57 & \text { SBD } & \text { Silicified, sparsely vesicular, aphyric, massive volcanic rock }\end{array}$

37Z-1, 6-7 $344.56 \quad 57$ SBD $\quad$ Silicified, sparsely vesicular, aphyric, massive volcanic rock

37Z-2, 18-20 $346.00 \quad 57 \quad$ SBD $\quad$ Silicified, sparsely vesicular, aphyric, massive volcanic rock

37Z-2, 31-33 $346.13 \quad 58 \quad$ SBD $\quad$ Magnetite-rich, clastic rock

37Z-2, 65-68 $346.47 \quad 59 \quad$ SBD $\quad$ Silicified, locally magnetite-bearing, aphyric volcanic rock

38Z-2, 90-92 $\quad 351.19 \quad 61 \quad$ SBD Completely altered, aphyric, amygdular volcanic rock

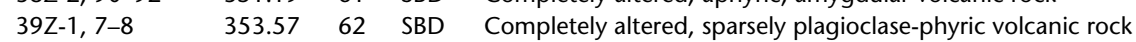

39Z-1, 41-43 $353.91 \quad 62$ SBD Completely altered, sparsely plagioclase-phyric volcanic rock

39Z-1, 83-87 $354.32 \quad 63$ HP Completely altered, silicified, aphyric volcanic rock

39Z-2, 18-19 $355.10 \quad 64 \quad$ SBD $\quad$ Completely altered, aphyric volcanic rock

40Z-1, 3-5 $358.03 \quad 65$ SBD Completely altered, magnetite-enriched, vesicular volcanic rock

40Z-1, 36-38 $358.36 \quad 66$ SBD Completely altered, sparsely plagioclase-phyric volcanic rock

40Z-2, 35-36 $359.71 \quad 67 \quad$ SBD $\quad$ Completely altered, clastic-textured volcanic rock

41Z-1, 46-47 $362.96 \quad 69$ SBD Completely altered, aphyric, amygdular volcanic rock

41Z-2, 7-10 $\quad 363.95 \quad 69$ SBD Completely altered, aphyric, amygdular volcanic rock

42Z-1, 30-31 $\quad 367.30 \quad 70 \quad$ SBD $\quad$ Completely altered, aphyric, sparsely amygdaloidal volcanic rock

42Z-1, 72-74 $367.72 \quad 70 \quad$ SBD $\quad$ Completely altered, aphyric, sparsely amygdaloidal volcanic rock
Plagioclase, quartz (illite, chlorite, pyrite, magnetite, "illite-bearing mixed-layer")

Quartz (chlorite, illite, "anhydrite, pyrite")

Quartz, plagioclase, illite (pyrite, chlorite "halite, rutile")

Quartz (plagioclase, pyrite, magnetite, illite, "anhydrite, chlorite")

Quartz (plagioclase, pyrite, chlorite, "illite, anhydrite, magnetite")

Quartz (anhydrite, illite, "pyrite, chlorite")

Quartz (anhydrite, illite, pyrite, "plagioclase")

Quartz, plagioclase (illite, "anhydrite, pyrite, magnetite, chlorite")

Quartz (plagioclase, illite, pyrite, anhydrite, chlorite)

Plagioclase (magnetite, illite, alunite, Fe-spinel, chlorite, pyrite, brucite)

Quartz (plagioclase, anhydrite, magnetite, "illite, chlorite, pyrite")

Quartz, plagioclase (illite, chlorite, anhydrite, "pyrite, magnetite")

Quartz (anhydrite, illite, "chlorite, plagioclase, pyrite")

Quartz (anhydrite, illite, chlorite, "pyrite")

Quartz, plagioclase (pyrite "anhydrite, chlorite")

Quartz (anhydrite, illite, chlorite, pyrite, plagioclase, illite-bearing mixed-layer)

Quartz (plagioclase, anhydrite, illite, chlorite, pyrite, magnetite)

Quartz (anhydrite, illite, pyrite, "plagioclase, chlorite")

Quartz (anhydrite, illite, pyrite, "plagioclase, chlorite")

Quartz (plagioclase, pyrite, illite, chlorite, "magnetite, anhydrite")

Quartz (plagioclase, pyrite, illite, chlorite, "magnetite, anhydrite")

Quartz (anhydrite, illite, "pyrite")

Quartz (anhydrite, chlorite, illite, pyrite, "plagioclase") 
Table T2 (continued).

\begin{tabular}{|c|c|c|c|c|c|}
\hline $\begin{array}{l}\text { Core, section, } \\
\text { interval }(\mathrm{cm})\end{array}$ & $\begin{array}{l}\text { Depth } \\
\text { (mbsf) }\end{array}$ & Unit & Lab & Description & XRD identification \\
\hline $43 Z-1,6-8$ & 371.56 & 71 & SBD & Completely altered, aphyric, sparsely vesicular volcanic rock & Quartz (illite, pyrite, chlorite, "anhydrite, plagioclase") \\
\hline $43 Z-1,21$ & 371.71 & 71 & HP & Completely altered, aphyric, sparsely vesicular volcanic rock; gray central part & Quartz, plagioclase (anhydrite, pyrite, chlorite, illite "halite, rutile") \\
\hline $43 Z-1,21$ & 371.71 & 71 & HP & $\begin{array}{l}\text { Completely altered, aphyric, sparsely vesicular volcanic rock; light gray margin of } \\
\text { piece (alteration halo) }\end{array}$ & Quartz, illite (anhydrite, pyrite "rutile") \\
\hline $43 Z-1,67-69$ & 372.17 & 72 & SBD & Completely altered, magnetite-bearing, aphyric volcanic rock & Quartz, plagioclase (chlorite, pyrite, magnetite, "anhydrite") \\
\hline 43Z-1, 90-91 & 372.40 & 72 & SBD & Completely altered, magnetite-bearing, aphyric volcanic rock & Quartz, plagioclase (chlorite, anhydrite, "pyrite, magnetite") \\
\hline $44 Z-1,85-86$ & 374.85 & 72 & SBD & Completely altered, magnetite-bearing, aphyric volcanic rock & Quartz, plagioclase (chlorite, anhydrite, "pyrite, magnetite") \\
\hline
\end{tabular}

Notes: $\mathrm{SBD}=$ shipboard data $\mathrm{RA}=$ postcruise data from $\mathrm{R}$. Asada, $\mathrm{HP}=$ postcruise data from $\mathrm{H}$. Paulick. XRD =X-ray diffraction. The terms major (outside parenthesis), minor (inside parenthesis), and trace (inside both parenthesis and quotation marks) are applied to XRD analyses as explained in "Hydrothermal Alteration," p. 8, in the "Explanatory Notes" chapter of Shipboard Scientific Party (2002) and do not imply quantitative abundances. 
Table T3. Minerals identified in fresh to completely altered whole rocks by XRD analysis, Hole 1189A. (See table notes. Continued on next page.)

\begin{tabular}{|c|c|c|c|c|c|}
\hline $\begin{array}{l}\text { Core, section, } \\
\text { interval }(\mathrm{cm})\end{array}$ & $\begin{array}{l}\text { Depth } \\
\text { (mbsf) }\end{array}$ & Unit & Lab & Description & XRD identification \\
\hline \multicolumn{6}{|l|}{ 193-1189A- } \\
\hline $1 \mathrm{R}-1,0-4$ & 0.02 & 1 & SBD & Fresh, moderately vesicular, aphyric dacite & Plagioclase, opaline silica (augite, quartz, spinel) \\
\hline $1 \mathrm{R}-1,12-17$ & 0.12 & 1 & HP & Fresh, moderately vesicular, aphyric dacite & Plagioclase, opaline silica/volcanic glass (augite) \\
\hline $2 \mathrm{R}-1,0-6$ & 9.70 & 2 & HP & Moderately altered, dotted, vesicular aphyric dacite & Plagioclase (cristobalite) \\
\hline $2 \mathrm{R}-1,34-35$ & 10.04 & 2 & SBD & Moderately to completely altered, vesicular aphyric dacite & Plagioclase, cristobalite (K-feldspar, pyrite, chlorite-bearing mixed-layer) \\
\hline $2 \mathrm{R}-1,77-78$ & 10.47 & 2 & SBD & Moderately to completely altered, vesicular aphyric dacite & Anhydrite (cristobalite, plagioclase, pyrite, chlorite) \\
\hline $2 \mathrm{R}-1,113-115$ & 10.83 & 4 & SBD & Hydrothermal breccia with completely altered volcanic clasts & Anhydrite, cristobalite (pyrite, chlorite-bearing mixed-layer, "illite") \\
\hline $2 \mathrm{R}-1,126-137$ & 10.97 & 4 & HP & Hydrothermal breccia with completely altered volcanic clasts; green clast & Anhydrite, cristobalite (K-feldspar, chlorite, illite "mixed-layer clays, halite, rutile") \\
\hline $2 \mathrm{R}-1,126-137$ & 10.97 & 4 & HP & Hydrothermal breccia with completely altered volcanic clasts; white clast & Cristobalite, K-feldspar, plagioclase (anhydrite, pyrite "chlorite?") \\
\hline $2 \mathrm{R}-1,126-137$ & 11.00 & 4 & HP & Hydrothermal breccia with completely altered volcanic clasts; green clast & Cristobalite (K-feldspar, chlorite, illite "pyrite, halite") \\
\hline $3 R-1,0-6$ & 19.40 & 5 & HP & Highly to completely bleached, vesicular aphyric dacite & Cristobalite, plagioclase (pyrite, chlorite "K-feldspar, halite") \\
\hline $3 R-1,6-10$ & 19.46 & 5 & SBD & Highly to completely bleached, vesicular aphyric dacite & Plagioclase, cristobalite (pyrite, chlorite) \\
\hline $3 R-1,14-17$ & 19.54 & 5 & $\mathrm{KL}$ & Highly to completely bleached, vesicular aphyric dacite & Cristobalite, plagioclase (quartz, chlorite, chlorite-bearing mixed-layer) \\
\hline $3 R-1,20-22$ & 19.60 & 5 & SBD & Highly to completely bleached, vesicular aphyric dacite & Cristobalite, plagioclase (K-feldspar, pyrite, chlorite, illite) \\
\hline $3 R-1,59-63$ & 19.99 & 6 & SBD & Hydrothermally fractured volcaniclastic breccia & Quartz, anhydrite (pyrite, "illite") \\
\hline $3 R-1,70-73$ & 20.10 & 7 & SBD & Bleached and silicified, intensely veined dacite & Quartz (K-feldspar, pyrite, "chlorite, illite") \\
\hline $3 R-1,84-89$ & 20.24 & 7 & HP & Bleached and silicified, intensely veined dacite & Quartz (K-feldspar, pyrite) \\
\hline $4 \mathrm{R}-1,14-17$ & 29.24 & 8 & $\mathrm{KL}$ & Completely altered dacite & Quartz, plagioclase (K-feldspar, chlorite, illite, "pyrite") \\
\hline $4 \mathrm{R}-1,17-20$ & 29.27 & 8 & SBD & Completely altered dacite & Quartz (plagioclase, pyrite, chlorite, illite) \\
\hline $4 \mathrm{R}-1,21-24$ & 29.31 & 8 & SBD & Completely altered dacite & Quartz (plagioclase, chlorite, pyrite, "illite") \\
\hline $5 R-1,14-16$ & 38.94 & 9 & SBD & Completely altered, hydrothermal breccia with flow-laminated clasts & Quartz (anhydrite, chlorite, pyrite, illite) \\
\hline $5 R-1,33-35$ & 39.1 & 9 & RA & Completely altered, hydrothermal breccia with flow-laminated clasts & Quartz, anhydrite, gypsum (chlorite, illite, "plagioclase") \\
\hline $5 R-1,41-50$ & 39.24 & 9 & HP & Completely, domainal, altered breccia with flow-laminated clasts; dominantly green & Quartz (anhydrite, chlorite, illite "plagioclase") \\
\hline $5 R-1,41-50$ & 39.24 & 9 & HP & Completely, domainal, altered breccia with flow-laminated clasts; dominantly white & Quartz, plagioclase (K-feldspar, chlorite, illite "pyrite") \\
\hline $6 \mathrm{R}-1,0-5$ & 48.6 & 9 & RA & Completely altered, hydrothermal breccia with flow-laminated clasts & Anhydrite, gypsum (quartz, chlorite, illite) \\
\hline $7 \mathrm{R}-1,4-12$ & 58.41 & 10 & HP & Completely altered, slightly vesicular volcanic rock & Quartz, plagioclase (K-feldspar, pyrite) \\
\hline $7 R-1,27-28$ & 58.57 & 10 & SBD & Completely altered, slightly vesicular volcanic rock & Quartz, plagioclase (pyrite, anhydrite, chlorite, illite) \\
\hline $7 \mathrm{R}-1,30-33$ & 58.60 & 10 & $\mathrm{KL}$ & Completely altered, slightly vesicular volcanic rock & Quartz, K-feldspar (plagioclase, rhodochrosite, chlorite, illite) \\
\hline $7 \mathrm{R}-1,58-59$ & 58.88 & 11 & SBD & Completely altered hydrothermal(?) breccia & Quartz (anhydrite, pyrite, "illite, chlorite") \\
\hline 7R-1, 95-96 & 59.25 & 13 & SBD & Completely altered hydrothermal(?) breccia & Quartz (plagioclase, chlorite, "pyrite, anhydrite") \\
\hline $8 \mathrm{R}-1,3-10$ & 68.04 & 15 & HP & Poikiloblastically silicified plagioclase-rich volcanic rock & Plagioclase, cristobalite (K-feldspar "quartz, pyrite, illite") \\
\hline $8 \mathrm{R}-1,31-32$ & 68.31 & 15 & SBD & Poikiloblastically silicified plagioclase-rich volcanic rock & Plagioclase (quartz, pyrite, "anhydrite, chlorite") \\
\hline $8 \mathrm{R}-1,39-41$ & 68.4 & 15 & RA & Volcanic rock with patchy silicification and prominent quartz-pyrite veining & Quartz, pyrite, plagioclase (cristobalite, chlorite, illite, mixed-layer clays) \\
\hline $8 \mathrm{R}-1,42-44$ & 68.42 & 15 & SBD & Poikiloblastically silicified plagioclase-rich volcanic rock & Plagioclase (quartz, K-feldspar, pyrite, "anhydrite, smectite, chlorite") \\
\hline $8 \mathrm{R}-1,90-92$ & 68.90 & 15 & SBD & Poikiloblastically silicified plagioclase-rich volcanic rock & Plagioclase, quartz (pyrite, K-feldspar, "anhydrite, chlorite, illite) \\
\hline $8 \mathrm{R}-1,113-114$ & 69.13 & 15 & SBD & Poikiloblastically silicified plagioclase-rich volcanic rock & Quartz, plagioclase (chlorite, pyrite") \\
\hline $9 \mathrm{R}-1,0-5$ & 77.70 & 16 & $\mathrm{KL}$ & Completely altered hydrothermal breccia & Quartz, plagioclase (anhydrite, chlorite, "illite, pyrite") \\
\hline $9 \mathrm{R}-1,16-17$ & 77.86 & 16 & SBD & Completely altered hydrothermal breccia & Quartz, anhydrite (plagioclase, pyrite, "chlorite") \\
\hline $9 \mathrm{R}-1,17-28$ & 77.91 & 16 & HP & Completely altered hydrothermal breccia & Quartz (plagioclase, anhydrite "pyrite, chlorite") \\
\hline $9 \mathrm{R}-1,63-67$ & 78.33 & 16 & $\mathrm{KL}$ & Completely altered hydrothermal breccia & Anhydrite, quartz (plagioclase, gypsum, pyrite, chlorite, "illite") \\
\hline $10 \mathrm{R}-1,0-1$ & 87.30 & 17 & SBD & Completely bleached moderately vesicular volcanic rock & Quartz (plagioclase, pyrite, "chlorite") \\
\hline $10 \mathrm{R}-1,38-51$ & 87.69 & 19 & HP & Completely altered hydrothermal breccia; gray-green coherent part & Quartz, plagioclase, K-feldspar (pyrite) \\
\hline 10R-1, 38-51 & 87.69 & 19 & HP & Completely altered hydrothermal breccia; clastic (siliaceous matrix and clasts) & Quartz (K-feldspar, anhydrite, pyrite, illite) \\
\hline $10 \mathrm{R}-1,38-51$ & 87.69 & 19 & HP & Completely altered hydrothermal breccia; green-gray mass & Quartz (K-feldspar, pyrite, chlorite, illite "anhydrite, halite, gypsum") \\
\hline $10 \mathrm{R}-1,38-51$ & 87.69 & 19 & $\mathrm{HP}$ & Completely altered hydrothermal breccia; gray-green mass & Quartz, plagioclase (K-feldspar, pyrite, chlorite "halite") \\
\hline $10 \mathrm{R}-1,50-51$ & 87.80 & 19 & SBD & Completely altered hydrothermal breccia & Quartz, anhydrite (plagioclase, pyrite, "chlorite, illite") \\
\hline 10R-1, 77-81 & 88.07 & 19 & $\mathrm{KL}$ & Completely altered hydrothermal breccia & Quartz, K-feldspar (plagioclase, pyrite, chlorite, "illite") \\
\hline 10R-1, 103-115 & 88.3 & 19 & RA & Completely bleached, moderately vesicular volcanic rock & Quartz, plagioclase (pyrite, gypsum, chlorite, illite, "mixed-layer clays") \\
\hline $11 \mathrm{R}-1,37-38$ & 97.27 & 20 & SBD & Completely silicified, massive, vesicular volcanic rock & Quartz (pyrite, illite, chlorite, "anhydrite") \\
\hline
\end{tabular}

Plagioclase, cristobalite (pyrite, chlorite)

Quartz, anhydrite (pyrite, "illite")

Quartz (K-feldspar, pyrite)

Quartz, plagioclase (K-feldspar, chlorite, illite, "pyrite")

Quartz (plagioclase, pyrite, chlorite, illite)

Quartz (plagioclase, chlorite, pyrite, "illite")

Quartz, anhydrite, gypsum (chlorite, illite, "plagioclase")

Quartz (anhydrite, chlorite, illite "plagioclase")

(

Quartz, plagioclase (pyrite, anhydrite, chlorite, illite)

Quartz, K-feldspar (plagioclase, rhodochrosite, chlorite, illite)

Quartz (anhydrite, pyrite, "illite, chlorite")

Plagioclase, cristobalite (K-feldspar "quartz, pyrite, illite")

Plagioclase (quartz, pyrite, "anhydrite, chlorite")

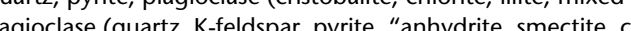

Qurtz, plagioclase (chlorite, pyrite")

uartz, plagioclase (anhydrite, chlorite, "illite, pyrite")

Quartz, anhydrite (plagioclase, pyrite, "chlorite")

Anhydrite, quartz (plagioclase, gypsum, pyrite, chlorite, "illite")

gioclase, pyrite, "chlorite")

Quartz (K-feldspar, anhydrite, pyrite, illite)

Quartz (K-feldspar, pyrite, chlorite, illite "anhydrite, halite, gypsum")

artz, plagioclase (K-feldspar, pyrite, chlorite "halite")

Quartz (pyrite, illite, chlorite, "anhydrite") 
Table T3 (continued).

\begin{tabular}{llllll}
\hline $\begin{array}{c}\text { Core, section, } \\
\text { interval (cm) }\end{array}$ & $\begin{array}{l}\text { Depth } \\
\text { (mbsf) }\end{array}$ & Unit & Lab & \multicolumn{1}{c}{ Description } & XRD identification \\
\hline $12 R-1,41-50$ & 106.9 & 20 & RA & Completely bleached, moderately vesicular volcanic rock & Quartz, pyrite, chalcopyrite (illite, "chlorite") \\
$12 R-1,69-70$ & 107.19 & 20 & SBD & Completely silicified, massive, vesicular volcanic rock & Quartz (illite, pyrite) \\
$12 R-1,83-86$ & 107.33 & 20 & KL & Completely silicified, massive, vesicular volcanic rock & Quartz (chlorite, illite, pyrite) \\
QR-1, $83-86$ & 107.33 & 20 & HP & Completely silicified, massive, vesicular volcanic rock & Quartz (chlorite, illite "pyrite, halite") \\
Quartz, illite ("anhydrite, pyrite") & Quartz (chlorite, pyrite, illite) \\
$12 \mathrm{R}-1,120-128$ & 107.70 & 21 & HP & Mineralized pumice breccia; separate of pumice fragments & Quartz (albite, pyrite, chlorite "illite") \\
$13 R-1,24-33$ & 116.34 & 22 & SBD & Pervasively bleached moderately vesicular volcanic rock & Quartz, chlorite (pyrite, illite "halite") \\
$13 R-1,51-53$ & 116.61 & 23 & HP & Polymict volcaniclastic breccia; bulk rock & Quartz (pyrite, chlorite, illite "halite") \\
$13 R-1,51-53$ & 116.61 & 23 & HP & Polymict volcaniclastic breccia; light green clast & Quartz (plagioclase, pyrite, chlorite, "illite") \\
$13 R-1,51-53$ & 116.61 & 23 & HP & Polymict volcaniclastic breccia; light gray clast & Quartz (pyrite, "chlorite, illite") \\
$13 R-1,51-53$ & 116.61 & 23 & SBD & Polymict volcaniclastic breccia & \\
$13 R-1,62-71$ & 116.72 & 24 & SBD & Pervasively bleached sparsely vesicular volcanic rock & \\
\hline
\end{tabular}

Notes: $\mathrm{SBD}=$ shipboard data, $\mathrm{HP}=$ postcruise data from $\mathrm{H}$. Paulick, $\mathrm{KL}=$ postcruise data from $\mathrm{K}$. Lackschewitz, RA = postcruise data from $\mathrm{R}$. Asada. $\mathrm{XRD}=\mathrm{X}$-ray diffraction. The terms majo (outside parenthesis), minor (inside parenthesis), and trace (inside both parenthesis and quotation marks) are applied to XRD analyses as explained in "Hydrothermal Alteration," p. 8, in the "Explanatory Notes" chapter of Shipboard Scientific Party (2002) and do not imply quantitative abundances. 
Table T4. Minerals identified in altered whole rocks by XRD analysis, Hole 1189B. (See table notes. Continued on next page.)

\begin{tabular}{|c|c|c|c|c|c|}
\hline $\begin{array}{l}\text { Core, section, } \\
\text { interval }(\mathrm{cm})\end{array}$ & $\begin{array}{l}\text { Depth } \\
\text { (mbsf) }\end{array}$ & Unit & Lab & Description & XRD identification \\
\hline \multicolumn{6}{|l|}{ 193-1189B- } \\
\hline $1 \mathrm{R}-1,0$ & 31.0 & & SBD & Hammer sample (for further explanation see text) & Pyrite (chalcopyrite, illite, "sphalerite") \\
\hline $1 \mathrm{R}-1,0-2$ & 31.0 & 1 & SBD & Semimassive sulfide with sparse volcanic rock clasts & Anhydrite (pyrite, chalcopyrite, gypsum) \\
\hline $1 \mathrm{R}-1,0-4$ & 31.0 & 1 & RA & Semimassive sulfide with sparse volcanic rock clasts & Anhydrite (gypsum, pyrite, chacopyrite, chlorite) \\
\hline $1 \mathrm{R}-1,20-30$ & 31.20 & 1 & SBD & Semimassive sulfide with sparse volcanic rock clasts & Pyrite, illite, chlorite/smectite-mixed-layer ("sphalerite") \\
\hline $1 \mathrm{R}-1,30-31$ & 31.30 & 2 & SBD & Completely altered, moderately vesicular, aphyric rock & Pyrite, muscovite (chlorite) \\
\hline $2 \mathrm{R}-1,11-20$ & 40.21 & 3 & SBD & $\begin{array}{l}\text { Breccia of completely altered volcanic rock clasts in a pyrite-anhydrite stockwork } \\
\text { matrix }\end{array}$ & Anhydrite (gypsum, pyrite) \\
\hline $2 \mathrm{R}-1,11-20$ & 40.21 & 3 & SBD & $\begin{array}{l}\text { Breccia of completely altered volcanic rock clasts in a pyrite-anhydrite stockwork } \\
\text { matrix }\end{array}$ & Gypsum, pyrite (anhydrite, illite) \\
\hline $2 \mathrm{R}-1,40-50$ & 40.50 & 3 & SBD & Pyrite-anhydrite stockwork matrix & Pyrite, anhydrite, quartz (gypsum) \\
\hline $2 \mathrm{R}-1,40-50$ & 40.50 & 3 & SBD & Wallrock sample & Pyrite (illite, chlorite, kaolinite?, "smectite, quartz") \\
\hline $3 R-1,0-10$ & 49.70 & 3 & SBD & $\begin{array}{l}\text { Breccia of completely altered volcanic rock clasts in a pyrite-anhydrite stockwork } \\
\text { matrix }\end{array}$ & Quartz, pyrite ("illite") \\
\hline $5 R-1,11-22$ & 69.41 & 4 & SBD & Breccia of completely altered volcanic rock clasts in a pyrite-quartz matrix & Pyrite (quartz, k-feldspar, illite, "chlorite") \\
\hline $5 R-1,45-55$ & 69.75 & 4 & $\mathrm{HP}$ & $\begin{array}{l}\text { Breccia of completely altered volcanic rock clasts in a pyrite-quartz matrix; separate of } \\
\text { volcanic clast }\end{array}$ & Quartz (K-feldspar, chlorite, illite "halite") \\
\hline $6 \mathrm{R}-1,0-12$ & 79.00 & 5 & HP & $\begin{array}{l}\text { Breccia of completely altered volcanic rock clasts in quartz-hematite-pyrite matrix; } \\
\text { separate of green volcanic clast }\end{array}$ & Chlorite (quartz, illite "K-feldspar, pyrite, halite") \\
\hline $6 \mathrm{R}-1,23-33$ & 79.23 & 5 & SBD & Breccia of completely altered volcanic rock clasts in quartz-hematite-pyrite matrix & Quartz (K-feldspar, plagioclase, pyrite, chlorite, "illite") \\
\hline $6 \mathrm{R}-1,64-74$ & 79.64 & 7 & SBD & Completely altered, aphyric volcanic rock & Quartz (plagioclase, K-feldspar, pyrite, chlorite, "illite") \\
\hline $7 \mathrm{R}-1,0-10$ & 88.70 & 8 & SBD & Breccia of completely altered volcanic rock clasts & Quartz, pyrite (K-feldspar, "illite") \\
\hline $8 \mathrm{R}-1,7-9$ & 98.47 & 9 & SBD & Completely altered, slightly vesicular, aphyric volcanic rock & K-feldspar (pyrite, chlorite, illite, "illite-bearing mixed-layer) \\
\hline 10R-1, 26-27 & 118.16 & 13 & SBD & Silica-clay altered, aphyric volcanic rock & Quartz (chlorite, K-feldspar, plagioclase, pyrite) \\
\hline $10 R-1,57-60$ & 118.47 & 14 & SBD & Silica-chlorite altered, spherulitic, flow-banded aphyric volcanic rock & Quartz (chlorite, K-feldspar, anhydrite, pyrite, illite) \\
\hline $10 \mathrm{R}-1,57-60$ & 118.47 & 14 & HP & Silica-chlorite altered, spherulitic, flow-banded aphyric volcanic rock; white bands & Chlorite (quartz, pyrite, illite "K-feldspar, halite") \\
\hline $10 \mathrm{R}-1,57-60$ & 118.47 & 14 & HP & Silica-chlorite altered, spherulitic, flow-banded aphyric volcanic rock; green bands & Chlorite (quartz "K-feldspar, pyrite, illite, halite") \\
\hline 10R-1, 67-69 & 118.57 & 15 & SBD & Completely altered, silicified, massive, aphyric volcanic rock & Cristobalite, plagioclase (K-feldspar, chlorite, "pyrite") \\
\hline $11 \mathrm{R}-1,0-7$ & 127.60 & 16 & SBD & Breccia of completely altered volcanic clasts & $\begin{array}{l}\text { Quartz (anhydrite, K-feldspar, chlorite, chlorite-bearing mixed-layer, smectite, illite, } \\
\text { pyrite) }\end{array}$ \\
\hline $11 \mathrm{R}-1,14-16$ & 127.74 & 17 & SBD & Jasperoidal breccia of completely altered volcanic clasts & Quartz (anhydrite, pyrite, "hematite") \\
\hline $11 \mathrm{R}-1,19-20$ & 127.79 & 18 & SBD & Polymict breccia of completely altered green volcanic clasts & Quartz (k-feldspar, illite, chlorite, pyrite, "plagioclase") \\
\hline $11 \mathrm{R}-1,22-27$ & 127.82 & 18 & HP & Polymict breccia of completely altered green volcanic clasts & Quartz (K-feldspar "anhydrite, pyrite") \\
\hline $11 \mathrm{R}-1,63-65$ & 128.23 & 19 & SBD & Completely altered, moderately vesicular, aphyric volcanic rock & $\begin{array}{l}\text { Cristobalite, plagioclase (quartz, "pyrite, chlorite, chlorite-bearing mixed-layer, } \\
\text { smectite") }\end{array}$ \\
\hline $11 \mathrm{R}-2,46-47$ & 128.74 & 19 & SBD & Completely altered, moderately vesicular, aphyric volcanic rock & Cristobalite, plagioclase (quartz, chlorite, "chlorite-bearing mixed-layer, smectite") \\
\hline $11 \mathrm{R}-2,66-76$ & 128.9 & 19 & RA & Very highly altered, moderately vesicular, aphyric volcanic rock & Cristobalite, plagioclase (quartz, smectite) \\
\hline $11 \mathrm{R}-2,89-91$ & 129.17 & 19 & SBD & Completely altered, moderately vesicular, aphyric volcanic rock & Cristobalite, plagioclase (quartz, smectite, "chlorite) \\
\hline $11 \mathrm{R}-3,0-29$ & 129.72 & 19 & $\mathrm{HP}$ & Completely altered, moderately vesicular, aphyric volcanic rock & Cristobalite, plagioclase (K-feldspar) \\
\hline $12 \mathrm{R}-1,114-115$ & 138.44 & 19 & SBD & Completely altered, moderately vesicular, aphyric volcanic rock & Plagioclase (K-feldspar, quartz, chlorite, "illite, pyrite") \\
\hline $12 \mathrm{R}-2,55-57$ & 139.26 & 19 & SBD & Completely altered, moderately vesicular, aphyric volcanic rock & $\begin{array}{l}\text { Cristobalite, plagioclase (K-feldspar, quartz, chlorite-bearing mixed-layer, "smectite, } \\
\text { illite") }\end{array}$ \\
\hline $13 R-1,22-24$ & 147.22 & 20 & SBD & Breccia with completely altered volcanic clasts & Quartz (plagioclase, pyrite, "chlorite") \\
\hline $13 R-1,34-36$ & 147.34 & 20 & SBD & Breccia with completely altered volcanic clasts & Quartz (pyrite, "barite") \\
\hline $13 \mathrm{R}-1,48$ & 147.48 & 21 & HP & Completely altered, massive, perlitic, aphyric volcanic rock & Quartz (K-feldspar, plagioclase "chlorite?, mixed layer clays?") \\
\hline $13 \mathrm{R}-1,52-54$ & 147.52 & 21 & SBD & Completely altered, massive, aphyric volcanic rock & Quartz (plagioclase, chlorite, illite, pyrite") \\
\hline $14 \mathrm{R}-1,0-10$ & 156.50 & 22 & SBD & Silicified and mineralized, aphyric volcanic rock & Quartz (pyrite, "illite") \\
\hline $14 \mathrm{R}-1,57-58$ & 157.07 & 23 & SBD & Breccia of completely altered volcanic rock fragments & Anhydrite, quartz ("plagioclase, chlorite") \\
\hline $14 \mathrm{R}-1,87$ & 157.37 & 23 & HP & Breccia of completely altered, flow-banded, volcanic rock fragments & Quartz, anhydrite (plagioclase, K-feldspar, chlorite, illite "pyrite") \\
\hline $14 \mathrm{R}-1,117-119$ & 157.67 & 23 & SBD & Breccia of completely altered volcanic rock fragments & Anhydrite, quartz (K-feldspar, plagioclase, chlorite) \\
\hline $14 \mathrm{R}-2,10-11$ & 157.89 & 23 & SBD & Breccia of completely altered volcanic rock fragments & Quartz (plagioclase, pyrite, "chlorite") \\
\hline
\end{tabular}

Pyrite (illite, chlorite, kaolinite?, "smectite, quartz")

Quartz, pyrite ("illite")

Chlorite (quartz, illite "K-feldspar, pyrite, halite")

Quartz (K-feldspar, plagioclase, pyrite, chlorite, "illite")

Quartz (chlorite, K-feldspar, plagioclase, pyrite)

Quartz (chlorite, K-feldspar, anhydrite, pyrite, illite)

Chlorite (quartz, pyrite, illite "K-feldspar, halite")

Cristobalite, plagioclase (K-feldspar, chlorite, "pyrite")

pyrite)

Cristobalite, plagioclase (quartz "pyrite, chlorite, chlorite-bearing mixed-layer, smectite"

Cristobalite, plagioclase (quartz, smectite)

Cristobalite, plagioclase (quartz, smectite, "chlorite)

Quartz (plagioclase, pyrite, "chlorite")

(K

uartz (plagioclase, chlorite, illite, pyrite")

Quartz (plagioclase, pyrite, "chlorite") 
Table T4 (continued).

\begin{tabular}{|c|c|c|c|c|c|}
\hline $\begin{array}{l}\text { Core, section, } \\
\text { interval }(\mathrm{cm})\end{array}$ & $\begin{array}{l}\text { Depth } \\
\text { (mbsf) }\end{array}$ & Unit & Lab & Description & XRD identification \\
\hline $14 \mathrm{R}-2,29-35$ & 158.08 & 24 & SBD & Completely altered, brecciated, silicified, aphyric volcanic rock & Quartz (pyrite, plagioclase, K-feldspar) \\
\hline $15 R-1,16-17$ & 166.26 & 25 & SBD & Breccia completely altered and silicified volcanic rock & $\begin{array}{l}\text { Cristobalite, plagioclase (K-feldspar, illite, chlorite, "smectite, chlorite-bearing mixed } \\
\text { layer, illite-bearing mixed-layer, pyrite") }\end{array}$ \\
\hline $15 \mathrm{R}-1,25$ & 166.35 & 25 & HP & Breccia completely altered and silicified, flow-banded, volcanic rock; large clast & Cristobalite, plagioclase (K-feldspar "chlorite, illite") \\
\hline $15 \mathrm{R}-1,25$ & 166.35 & 25 & $\mathrm{HP}$ & $\begin{array}{l}\text { Breccia completely altered and silicified, flow-banded, volcanic rock; groundmass + } \\
\text { small clasts }\end{array}$ & Quartz, cristobalite, plagioclase (K-feldspar, pyrite, chlorite, illite) \\
\hline $15 R-1,124$ & 167.34 & 25 & $\mathrm{HP}$ & Breccia completely altered and silicified, flow-banded, volcanic rock; green clast & Cristobalite (plagioclase, pyrite, chlorite "illite, K-feldspar") \\
\hline $15 R-1,124$ & 167.34 & 25 & HP & Breccia completely altered and silicified, flow-banded, volcanic rock; gray clast & Cristobalite (quartz, K-feldspar, plagioclase, chlorite, illite "pyrite, halite") \\
\hline $15 R-1,128-129$ & 167.38 & 25 & SBD & Breccia completely altered and silicified volcanic rock & $\begin{array}{l}\text { Cristobalite, plagioclase (K-feldspar, illite, chlorite, "smectite, chlorite-bearing mixed } \\
\text { layer, illite-bearing mixed-layer, pyrite") }\end{array}$ \\
\hline $15 R-2,61-64$ & 168.21 & 26 & SBD & Silicified, sparsely vesicular, aphyric volcanic rock & $\begin{array}{l}\text { Cristobalite, plagioclase (K-feldspar, illite, "chlorite, smectite, chlorite-bearing mixed } \\
\text { layer, pyrite) }\end{array}$ \\
\hline $16 \mathrm{R}-1,36-39$ & 176.06 & 26 & SBD & Silicified, sparsely vesicular, aphyric volcanic rock & $\begin{array}{l}\text { Cristobalite, plagioclase (K-feldspar, "illite, illite-bearing mixed-layer, chlorite, } \\
\text { smectite, chlorite-bearing mixed-layer) }\end{array}$ \\
\hline $16 \mathrm{R}-1,74-81$ & 176.45 & 27 & HP & Very highly altered, moderately vesicular, aphyric volcanic rock & Quartz (K-feldspar, plagioclase "pyrite") \\
\hline $16 \mathrm{R}-1,130-131$ & 177.00 & 27 & SBD & Very highly altered, moderately vesicular, aphyric volcanic rock & Quartz (plagioclase, pyrite, chlorite-bearing mixed-layer) \\
\hline $16 \mathrm{R}-2,9-10$ & 177.22 & 27 & SBD & Very highly altered, moderately vesicular, aphyric volcanic rock & Quartz (plagioclase, K-feldspar, chlorite-bearing mixed-layer, "pyrite, illite") \\
\hline $17 R-1,19-20$ & 185.49 & 28 & SBD & Silicified, moderately vesicular, aphyric volcanic rock & Quartz (plagioclase, K-feldspar, pyrite) \\
\hline $17 \mathrm{R}-1,56-60$ & 185.87 & 30 & $\mathrm{HP}$ & Silicified, flow-banded volcanic rock & Quartz, plagioclase (K-feldspar, pyrite) \\
\hline $17 \mathrm{R}-1,60-63$ & 185.90 & 30 & SBD & Silicified, flow-banded volcanic rock & Quartz (plagioclase, K-feldspar, pyrite, chlorite-bearing mixed-layer) \\
\hline $17 R-1,65-66$ & 189.95 & 30 & SBD & Silicified, flow-banded volcanic rock & Quartz (plagioclase, K-feldspar, pyrite, chlorite-bearing mixed-layer) \\
\hline $17 \mathrm{R}-1,96-98$ & 186.26 & 31 & SBD & Normal graded, thinly bedded, volcaniclastic sandstone & Quartz (plagioclase, pyrite, illite) \\
\hline $17 R-1,118-122$ & 186.48 & 32 & SBD & Flow-banded silicified volcanic rock & $\begin{array}{l}\text { Quartz (plagioclase, K-feldspar, pyrite, chlorite, "illite, illite-bearing mixed-layer, } \\
\text { chlorite/smectite mixed-layer) }\end{array}$ \\
\hline $18 \mathrm{R}-1,4-12$ & 195.05 & 32 & HP & $\begin{array}{l}\text { Breccia of completely altered, flow-banded volcanic rock; light gray clasts + silica } \\
\text { matrix }\end{array}$ & Quartz, cristobalite, plagioclase (K-feldspar "pyrite") \\
\hline $18 \mathrm{R}-1,4-12$ & 195.05 & 32 & HP & $\begin{array}{l}\text { Breccia of completely altered, flow-banded volcanic rock; dark green clasts + silica } \\
\text { matrix }\end{array}$ & Quartz, plagioclase (K-feldspar "pyrite") \\
\hline $18 \mathrm{R}-1,24-25$ & 195.24 & 33 & SBD & Breccia of completely altered, flow-banded volcanic rock & $\begin{array}{l}\text { Cristobalite, plagioclase (quartz, K-feldspar, illite, chlorite, (illite-bearing mixed-layer, } \\
\text { pyrite") }\end{array}$ \\
\hline $18 \mathrm{R}-1,45-50$ & 195.45 & 34 & HP & Breccia of completely altered flow-banded volcanic rock; spherulitic clast & K-feldspar (cristobalite, plagioclase "chlorite") \\
\hline $18 \mathrm{R}-1,45-50$ & 195.45 & 34 & $\mathrm{HP}$ & $\begin{array}{l}\text { Breccia of completely altered flow-banded volcanic rock; groundmass (small clasts + } \\
\text { siliceous matrix) }\end{array}$ & Quartz (cristobalite, K-feldspar, plagioclase "pyrite") \\
\hline $18 \mathrm{R}-1,70-71$ & 195.70 & 34 & SBD & Breccia of completely altered flow-banded volcanic rock & $\begin{array}{l}\text { Cristobalite, plagioclase (quartz, K-feldspar, pyrite, illite, chlorite, chlorite-bearing } \\
\text { mixed-layer, "illite-bearing mixed-layer, talc?") }\end{array}$ \\
\hline $18 \mathrm{R}-1,107-108$ & 196.07 & 34 & SBD & Breccia of completely altered flow-banded volcanic rock & $\begin{array}{l}\text { Cristobalite, plagioclase (quartz, K-feldspar, pyrite, illite, chlorite-bearing mixed- } \\
\text { layer, "smectite, illite-bearing mixed-layer, actinolite?") }\end{array}$ \\
\hline 18R-1, 124-131 & 196.25 & 34 & HP & Breccia of completely altered flow-banded volcanic rock; bulk rock & Cristobalite, plagioclase (K-feldspar "chlorite") \\
\hline $18 \mathrm{R}-1,124-131$ & 196.25 & 34 & $\mathrm{HP}$ & Breccia of completely altered flow-banded volcanic rock; light gray-green clast & Cristobalite (plagioclase, chlorite "tridymite, K-feldspar, illite") \\
\hline $18 \mathrm{R}-2,0-4$ & 196.42 & 34 & $\mathrm{HP}$ & Breccia of completely altered flow-banded volcanic rock & Cristobalite, plagioclase (quartz, K-feldspar "chlorite, illite") \\
\hline $18 \mathrm{R}-2,23-24$ & 196.65 & 34 & SBD & Breccia of completely altered flow-banded volcanic rock & $\begin{array}{l}\text { Cristobalite, plagioclase (quartz, K-feldspar, pyrite, illite, chlorite, chlorite-bearing } \\
\text { mixed-layer, "smectite, illite-bearing mixed-layer, actinolite?") }\end{array}$ \\
\hline $18 R-2,42-43$ & 196.84 & 35 & SBD & Completely altered, clast-supported polymict breccia & Quartz (plagioclase, K-feldspar, chlorite, "illite, pyrite") \\
\hline $18 \mathrm{R}-2,48-59$ & 196.91 & 35 & HP & Completely altered, clast-supported polymict breccia; spherulitic clast & Cristobalite (K-feldspar, plagioclase, chlorite, illite "tridymite, pyrite, halite") \\
\hline $18 \mathrm{R}-2,48-59$ & 196.91 & 35 & $\mathrm{HP}$ & Completely altered, clast-supported polymict breccia; flow-banded clast & Plagioclase (quartz, K-feldspar, chlorite, illite "pyrite, halite") \\
\hline $18 \mathrm{R}-2,48-59$ & 196.91 & 35 & $\mathrm{HP}$ & Completely altered, clast-supported polymict breccia; average groundmass & Plagioclase, quartz (K-feldspar "pyrite") \\
\hline $18 \mathrm{R}-2,48-59$ & 196.91 & 35 & HP & Completely altered, clast-supported polymict breccia; small, green clast & Quartz (plagioclase, K-feldspar, chlorite, illite "halite") \\
\hline
\end{tabular}

Notes; $\mathrm{SBD}=$ shipboard data, $\mathrm{RA}=$ postcruise data from R. Asada, HP = postcruise data from H. Paulick. XRD = X-ray diffraction. The terms major (outside parenthesis), minor (inside parenthesis), and trace (inside both parenthesis and quotation marks) are applied to XRD analyses as explained in "Hydrothermal Alteration," p. 8, in the "Explanatory Notes" chapter of Shipboard Scientific Party (2002) and do not imply quantitative abundances. 